Fundamentals of ICF Hohlraums

M. D. Rosen

October 4, 2005

Lectures presented at the Scottish Universities Summer School in Physics St. Andrews, United Kingdom August 15, 2005 through August 26, 2005 
This document was prepared as an account of work sponsored by an agency of the United States Government. Neither the United States Government nor the University of California nor any of their employees, makes any warranty, express or implied, or assumes any legal liability or responsibility for the accuracy, completeness, or usefulness of any information, apparatus, product, or process disclosed, or represents that its use would not infringe privately owned rights. Reference herein to any specific commercial product, process, or service by trade name, trademark, manufacturer, or otherwise, does not necessarily constitute or imply its endorsement, recommendation, or favoring by the United States Government or the University of California. The views and opinions of authors expressed herein do not necessarily state or reflect those of the United States Government or the University of California, and shall not be used for advertising or product endorsement purposes. 


\title{
Fundamentals of ICF Hohlraums
}

Mordecai D. ("Mordy") Rosen

\section{Lawrence Livermore National Laboratory (LLNL)}

\section{Lectures presented at the Scottish Universities Summer School in Physics}

\author{
St. Andrews, Aug. 15-26, 2005
}

\section{Lecture I: Basic Physics}

\section{Introduction to Lecture I:}

On the Nova Laser at LLNL, we demonstrated many of the key elements required for assuring that the next laser, the National Ignition Facility (NIF) will drive an Inertial Confinement Fusion (ICF) target to ignition. The indirect drive (sometimes referred to as "radiation drive" ) approach converts laser light to x-rays inside a gold cylinder, which then acts as an x-ray "oven" (called a hohlraum) to drive the fusion capsule in its center. On Nova we've demonstrated good understanding of the temperatures reached in hohlraums and of the ways to control the uniformity with which the x-rays drive the spherical fusion capsules. In these lectures we will be reviewing the physics of these laser heated hohlraums, recent attempts at optimizing their performance, and then return to the ICF problem in particular to discuss scaling of ICF gain with scale size, and to compare indirect vs. direct drive gains.

In ICF, spherical capsules containing Deuterium and Tritium (DT)-the heavy isotopes of hydrogen- are imploded, creating conditions of high temperature and density similar to those in the cores of stars required for initiating the fusion reaction. When DT fuses an alpha particle (the nucleus of a helium atom) and a neutron are created releasing large amounts of energy. If the surrounding fuel is sufficiently dense, the alpha particles are stopped and can heat it, allowing a selfsustaining fusion burn to propagate radially outward and a high gain fusion microexplosion ensues.

To create those conditions the outer surface of the capsule is heated (either directly by a laser or indirectly by laser produced $x$-rays) to cause rapid ablation and outward expansion of the capsule material. A rocket-like reaction to that 
outward flowing heated material leads to an inward implosion of the remaining part of the capsule shell. The pressure generated on the outside of the capsule can reach nearly 100 megabar (100 million times atmospheric pressure $\left[1 \mathrm{~b}=10^{6} \mathrm{cgs}\right]$ ), generating an acceleration of the shell of about 10 trillion gees, and causing that shell to reach, over the course of a few nanoseconds, an implosion velocities of 300 $\mathrm{km} / \mathrm{sec}$. When the shell and its contained fuel stagnates upon itself at the culmination of the implosion, most of the fuel is in a compressed shell which is at 1000 times solid density. That shell surrounds a hot spot of fuel with sufficient temperature (roughly $10 \mathrm{keV}$ or 100 million degrees) to ignite a fusion reaction.

The capsule must not only be driven hard, but also uniformly over its entire surface to cause uniform compression of the fuel to the center. With direct drive, this uniform heating of the capsule is caused by simultaneously illuminating the capsule from all sides with many laser beams and taking great care (via beam conditioning to avoid speckle etc.) to assure that 2 points close to one another on the capsule surface are driven with the same illumination. With indirect drive, the capsule is positioned in the center of a cylindrically symmetric container called a hohlraum. Laser beams enter the hohlraum through holes in the end caps, heat the walls of the cylinder, which then radiate soft $x$ rays, filling the hohlraum with a bath of radiant energy. This energy causes the fuel capsule to implode. Typically, $70-80 \%$ of the laser energy can be converted to $x$-rays. The hohlraum concept leads to a natural, geometric uniformity of $x$-ray flux on the capsule surface, since two points close to one another on the capsule surface "look out" at the heated hohlraum walls and (for a wall to capsule radius ratio of order 4) see nearly identical large sections of the walls (thus making it irrelevant just how non-uniform those sections actually are) and hence a nearly identical heat environment and are thus driven nearly identically. We now proceed to study these hohlraums. 


\section{History of hohlraums}

The year this lecture is given, 2005, has been designated "World Year of Physics" to celebrate the centenary of Einstein's 1905 publication of 4 "miraculous" papers: "photo-electric" effect, Brownian motion, special relativity, and " $\mathrm{E}=\mathrm{mc}^{2}$ ". Interestingly, despite all the revolutionary implications of special relativity (and later of general relativity) Einstein's 1923 Nobel Prize cites the first paper- the photoelectric effect. Its role in the development of hohlraum physics is what concerns us here. So let us take a further step (or two!) back in time:

Human beings have been using "hohlraums" for millennia, in the form of kilns - hot ovens that can harden clay pots. Their role in furthering human civilization through engendering storage, transportation, trade, and writing is well known. Jumping ahead to the late $19^{\text {th }}$ century, scientific inquiry into the nature of these ovens matured. As we shall see, an important figure of merit for any system near thermal equilibrium is its "optical depth" $\left(\tau_{\mathrm{O} . \mathrm{D}}\right)$ - namely the ratio of a system length $\mathrm{L}$ to a typical mean free path ("m.f.p"). For a kiln a m.f.p. of a typical photon inside the walls is about $0.1 \mu \mathrm{m}$. The heated depth of the wall is about $1 \mathrm{~cm}$. Hence the walls of a kiln have an enormous $\tau_{\text {O.D. }}$ of about $10^{5}$ and thus a kiln is expected to be very close to equilibrium.

The spectrum of the electro-magnetic (EM) radiation emerging from a small hole of a hot $\left(1000-2000^{\circ}\right)$ oven was measured and turned out to be independent of wall material. Kirchoff also formulated his famous law that in equilibrium emissivity = absorptivity, so that the best absorbers make the best emitters. Thus a "black-body" whose name (in the optical range of photons) implies complete absorption (like an oven with only a small hole), will radiate more than any other system at the same temperature $\mathrm{T}$. This universal blackbody (BB) spectrum (emerging intensity [power per unit area per unit solid angle] $I_{v}$ within a small interval of frequency $d v$ vs. $v$ ) was measured and found to have the following shape: for small $v$, it rose with frequency as $v^{2} \mathrm{~T}$ (The so-called Rayleigh - Jeans (R-J) fit). It reached a peak (at $v=2.8 \mathrm{~T}$ when appropriate units are used, to be discussed 
below). Then it decayed as $v^{3} \mathrm{e}^{(-v / T)}$ (called the Wien fit). The quantity $I_{v}$ is related to the isotropic energy density $U_{v}$ by $I_{v}=\mathrm{cU}_{v} / 4 \pi$ and thus both have the same spectrum.

Besides this experimental work, Wien's law, derived from purely thermodynamic arguments, declared that this spectrum must be in the form of $\mathrm{U}_{v}=v^{3} \mathrm{f}(v / T)$. Note that Wien's fit clearly obeys his law, as does the R-J fit with $f(x)=1 / x$. An immediate consequence of Wien's law is that the total energy density which is an integral over $v$, will be $U=a T^{4}$. This gives a total $I=(\sigma / \pi) T^{4}$, where $\sigma=\mathrm{ac} / 4$. If we ask what the one sided flux $\mathrm{F}$ out the small hole will be, in a direction (we'll call $\mathrm{x}$ ) perpendicular to the plane of the hole we must do an integral over $\mu$ of I times $2 \pi \mu$ (where $\mu$ is the cosine of the angle between a ray and the $x$ axis) from $\mu=0$ to 1 , and get the well known $\mathrm{F}=\sigma \mathrm{T}^{4}$.

Planck set out to derive this spectrum. Based on EM theory he found that within a given frequency interval $\mathrm{d} v$, an oscillator of mass $\mathrm{m}$, absorbs energy as $\left(\pi \mathrm{e}^{2} / 3 \mathrm{~m}\right) \mathrm{U}_{v}$ where $U_{v}$ is the (sought after) frequency behavior of the energy density of the radiation. He set that equal to the emission from that radiator (since we're in equilibrium the oscillators in the hohlraum wall emit as much as they absorb) which, again from EM theory is $\left(8 \pi^{2} e^{2} v^{2} / 3 \mathrm{mc}^{3}\right) \mathrm{E}_{\text {osc }}$ where $\mathrm{E}_{\text {osc }}$ is the energy of the oscillator. Thus:

$$
\mathrm{U}_{v}=\left(8 \pi v^{2} / \mathrm{c}^{3}\right) \mathrm{E}_{\mathrm{osc}}
$$

Planck then put forward some not-unreasonable arguments for the entropy of a many oscillator state, that resulted in an expression: $\mathrm{d}^{2} \mathrm{~S} / \mathrm{dE}^{2}=-1 / v E$. That combined with the classical thermodynamic relation $\mathrm{dS} / \mathrm{dE}=1 / \mathrm{T}$, his Eq. (1.1), and Wien's law immediately resulted triumphantly in Wien's fit. But the triumph was short lived. Most of the data was in the large $v$, Wien's fit regime (for a $1500^{\circ}$ oven, the spectral peak will be at about a half of an $\mathrm{eV}$, so the optical range (IR to UV of 1$3 \mathrm{eV}$ where most instruments were available) is well past the peak. As technology progressed the far IR (low $v$ ) regime began to give up its secrets, and the Wien fit did not fit so well anymore!

With the R-J law emerging from the new data, Planck noticed that $\mathrm{d}^{2} \mathrm{~S} / \mathrm{dE}^{2}=$ $-1 / \mathrm{E}^{2}$ led, as above, to the R-J fit. He was thus quickly led to the form 
$\mathrm{d}^{2} \mathrm{~S} / \mathrm{dE}^{2}=-1 /(v+E) E$ as a way to possibly cover the whole spectrum. So now, $\mathrm{dS} / \mathrm{dE}=1 / \mathrm{T}$ leads to (and inserting 2 constants $\mathrm{h} \& \mathrm{k}$ that assure proper dimensionality within the exponential, as well as a good numerical fit to the R-J regime):

$$
\mathrm{E}_{\mathrm{osc}}=\mathrm{h} v /\left(\mathrm{e}^{(\mathrm{hv} / \mathrm{kT})}-1\right)
$$

And via Eq. (1.1) to:

$$
\mathrm{U}_{v \mathrm{BB}}=\left(8 \pi v^{2} / \mathrm{c}^{3}\right) \mathrm{hv} /\left(\mathrm{e}^{(\mathrm{hv} / \mathrm{kT})}-1\right)
$$

This successful fit only begged the question- what was the theoretical justification for such a form of the entropy? Planck spent the next " 2 most difficult months of my life" answering that question. Part of the difficulty was his necessity to adopt an approach he previously rejected- Boltzmann's probabilistic view of entropy as $S=k \ln W$, where $\mathrm{W}$ measures the probability of being in a microstate whose energy corresponds to the macro-state whose entropy we seek, along with the notion that all microstates are equally probable. Thus we need to calculate the multiplicity of states to find S. Following in the path of an earlier calculation of Boltzmann, Planck considered a system with $\mathrm{N}$ oscillators. He assigned an energy to each, but only in artificially assumed integer units ("quanta") of size $\Delta$. If the total energy of the system is $\mathrm{P} \Delta$, then the average energy of an oscillator will be $\mathrm{E}=\mathrm{P} \Delta / \mathrm{N}$.

Now for the combinatorics (which required the artificial integer assumption in order to proceed): A typical possibility of what the system could be, can be pictured / written as: "| $\ldots|.| \ldots \ldots|| \ldots|$.$| etc." Here we have assigned some$ number of energy units $\Delta$ (the dots) to each oscillator (the partitions I). If we consider this entire accounting enterprise as filling in $\mathrm{N}+\mathrm{P}$ blanks with $\mathrm{P}$ dots and $\mathrm{N}$ partitions, there are $(\mathrm{N}+\mathrm{P})$ ! ways to do so and we divide by $\mathrm{N} ! \mathrm{P}$ ! to avoid "double" counting choices that are the same but written in different order. This is what " $W$ " represents in $S=k \ln W$. Using Stirling's approximation, defining $x=$ $\mathrm{P} / \mathrm{N}=\mathrm{E} / \Delta$ we find $\mathrm{S} / \mathrm{k}=\{(1+\mathrm{x}) \ln (1+\mathrm{x})-\mathrm{x} \ln \mathrm{x}\}$ and then via $\mathrm{dS} / \mathrm{dE}=1 / \mathrm{T}$ we get $\left.\mathrm{E}=\Delta /\left(\mathrm{e}^{(\Delta / \mathrm{kT})}-1\right)\right)$. Then Wien's law forces $\Delta=\mathrm{h} v$ and the derivation is complete.

Planck stopped there (with finite, quantized $\Delta$ ) because it gave the right answer. Had he followed Boltzmann he would have taken $\Delta$ "classically" to zero and "derived" E=kT and thus via his Eq. (1.1) the R-J fit. Planck assumed that other 
workers would figure out the microphysics of oscillators and the meaning of these quanta. He spent over a decade trying to minimize the implications of this radical notion. Planck's paper was quite tersely written and difficult to follow, and remained rather neglected for 6 years. This sets the stage for 1905 and Einstein's "photo-electric" paper.

Einstein was troubled (as he would be throughout his career) with the lack of unity in physics- mechanics dealt with particles but EM theory dealt with waves. He interpreted Planck's work as still being in the classical camp (after all Planck's Eq. 1.1 is entirely from EM wave theory) so he mostly ignored him. Einstein did use Eq. 1.1 to immediately derive the R-J law (to him E=kT was obvious) and to point out the "ultra-violet" catastrophe- that the R-J law, if assumed to hold for all v, would make U diverge.

Einstein concentrated on the volume $(\mathrm{V})$ dependence of the entropy $\mathrm{S}$ of the radiation field in a hohlraum. He defined $S_{v} d v=V f\left(U_{v}\right) d v$ and of course $E_{E M v} d v=$ $\mathrm{VU}_{v} \mathrm{~d} v$. He then just inverted the Wien fit to find $-(\mathrm{k} / \mathrm{h} v) \ln \left(\mathrm{U}_{v} / \alpha v^{3}\right)=1 / \mathrm{T}$. He then set that equal to $d S / d E$ (sounds familiar?!) which is equal to $d f / d U_{v}$. This gives $f=-$ $\left(k U_{v} / h v\right)\left[\ln \left(U_{v} / \alpha v^{3}\right)-1\right]$, which gives $S_{v} d v=\left(\mathrm{k} \mathrm{E}_{v} / h v\right)\left[\ln \left(\mathrm{E}_{v} / \mathrm{V} v^{3} \mathrm{~d} v\right)-1\right]$. He then considered a change in volume from $\mathrm{V}_{0}$ to $\mathrm{V}$, and the ensuing change in entropy:

$$
\mathrm{S}_{\mathrm{BB}}-\mathrm{S}_{0}=\left(\mathrm{kE}_{\mathrm{v}} / \mathrm{hv}\right) \ln \left(\mathrm{V} / \mathrm{V}_{0}\right)
$$

and compared it to an $\mathrm{N}$ particle classical ideal gas result:

$$
\mathrm{S}_{\mathrm{ig}}-\mathrm{S}_{0}=(\mathrm{kN}) \quad \ln \left(\mathrm{V} / \mathrm{V}_{0}\right)
$$

Einstein then reminded us what Eq. (1.5) means in the $S=k \ln W$ context. The probability a localized particle will find itself in a volume $\mathrm{V}$ is $\left(\mathrm{V} / \mathrm{V}_{0}\right)$, and that $\mathrm{N}$ of them will is $\mathrm{W}=\left(\mathrm{V} / \mathrm{V}_{0}\right)^{\mathrm{N}}$. Comparing Eqs. (1.4) to (1.5) immediately told Einstein that light of energy $E_{v}$ behaves as if it is made up of $N$ localized particles (quantized photons), each with energy hv.

It is only the next year, 1906, when Einstein turned his attention to calculating the $\mathrm{T}, v$ behavior of $\mathrm{S}$, that he understood enough of what Planck did to see the profound connection to his 1905 photon paper, namely: Planck's quantized oscillators emit Einstein's quantized photons- and thus Quantum Mechanics (QM) is born! 
Since the notion of photons (vs. EM waves) proved far too radical for nearly everyone, further " hohlraum-inspired" developments also prove crucial. In 1907 Einstein reasoned that the vibrating molecules in a wall also radiate (far IR) and as oscillators should follow Eqs. (1.1) \& (1.2). So he took dE / dT via Eq. (1.2) to find the specific heat and to predict it vanishing as $\mathrm{T}$ approaches zero. A wide community of physicists was quite interested in this, measured the prediction to be true, and QM became accepted. Planck still resisted for another decade, but eventually gave in, and in 1919 won the Nobel Prize for ...QM!

It is not until 1917 that Einstein developed a full theory of photon-matter interaction. The less well-known part of that paper deals with how atoms in motion equilibrate with a photon field via momentum conserving interactions that prove the photon concept. This calculation is a forerunner (by 60 years!) of the lasercooling concept. The better-known part of the paper deals with equilibrating atoms at rest (whose excited states are Maxwell-Boltzmann (MB) distributed in thermal equilibrium) with the photon field via energy conserving interactions. This is a forerunner (by 45 years) of the laser concept. In it Einstein derives the Planckian (Eq. (1.3)) in 3 lines! In a two state atom (with energy levels $1 \& 2$ ) he balances (spontaneous \& stimulated) emission with absorption:

$$
\left(\mathrm{A}_{21}+\mathrm{B}_{21} \mathrm{I}_{\mathrm{v}}\right) \mathrm{N}_{2}=\mathrm{B}_{12} \mathrm{I}_{\mathrm{v}} \mathrm{N}_{1}
$$

along with the $\mathrm{MB}$ distribution requirement:

$$
\mathrm{N}_{2} / \mathrm{N}_{1}=\mathrm{e}^{-(\mathrm{E} 21 / \mathrm{kTT})}
$$

Solve this for $\mathrm{I}_{v}$ (along with the high $\mathrm{T}$ limit forcing $\mathrm{B}_{21}=\mathrm{B}_{12}$ ) and get:

$$
\mathrm{I}_{\mathrm{BBv}}=(\mathrm{A} / \mathrm{B}) /\left(\mathrm{e}^{(\mathrm{E} 21 / \mathrm{kT})}-1\right)
$$

Wien's law forces $E_{21}=h v$ and $(A / B)=v^{3}$ and then the constants are derived by having $\mathrm{I}_{\mathrm{BBv}}$ be equal to the R-J fit in the classical low $v$ regime. The Planckian is thus derived. Another important outgrowth of this treatment, which is a perfect lead-in to our next section, is a useful form of Kirchoff's law: The spontaneous emission "source term" $J_{v}=A_{21} N_{2}$ is, by Eq. (1.6), related to the net absorption by:

$$
\mathrm{J}_{v}=\mathrm{A}_{21} \mathrm{~N}_{2}=\mathrm{B}\left(\mathrm{N}_{1}-\mathrm{N}_{2}\right) \mathrm{I}_{\mathrm{BBv}}=\kappa^{\prime} \mathrm{I}_{\mathrm{BBv}} \text {. }
$$

Since $\mathrm{J}_{v}$ is a property of the atom, if the atom is in LTE (collisionally thermalized levels) this "source term" result will hold for other $I_{v}$ choices. We now turn our 
attention to precisely that situation, where $I_{v}$ is not exactly the Planckian, and find how it propagates through matter.

\section{Radiation transport}

We now ask the question: What if the radiation / matter were not exactly in equilibrium, but nearly so? Consider the gain and loss of a beam of photons as it propagates along its trajectory " $\mathrm{s}$ ".

$$
\mathrm{dI}_{v} / \mathrm{ds}=\mathrm{J}_{v}-\kappa^{\prime} \mathrm{I}_{v}=\kappa^{\prime}\left(\mathrm{I}_{\mathrm{BBv}}-\mathrm{I}_{v}\right)
$$

Here for simplicity we neglect many things (such as scattering, higher order terms such as time derivatives, etc) that are treated at great length in textbooks. To make progress easier (indeed to reduce the problem to its core physics) we define an optical depth coordinate:

$$
\tau_{v}(\mathrm{~L})=\int_{0}^{\mathrm{L}} \kappa^{\prime}{ }_{v} \mathrm{ds}
$$

This then allows us to re-write Eq. (1.10) simply as

$$
\mathrm{dI}_{\mathrm{v}} / \mathrm{d} \tau_{\mathrm{v}}=\mathrm{I}_{\mathrm{BBv}}-\mathrm{I}_{\mathrm{v}}
$$

Use a standard integrating factor $\exp \left(\tau_{v}\right)$ technique, integrate from $\tau_{v}(0)=0$ to $\tau_{v}(\mathrm{~L})$ and get:

or:

$$
\mathrm{I}_{v}(\mathrm{~L}) e^{\tau_{v}(\mathrm{~L})}-\mathrm{I}_{v 0}=\int_{\tau_{v}(0)}^{\tau_{v}(\mathrm{~L})} \mathrm{I}_{v \mathrm{~B}} e^{\tau_{v}} \mathrm{~d} \tau_{v}
$$

$$
\mathrm{I}_{v}(\mathrm{~L})=\int_{\tau_{v}(0)}^{\tau_{v}(\mathrm{~L})} I_{\nu \mathrm{B}} e^{\left(\tau_{v}-\tau_{v}(\mathrm{~L})\right)} \mathrm{d} \tau_{v}+\mathrm{I}_{0 v} e^{-\tau_{v}(\mathrm{~L})}
$$

From this 2 lessons emerge immediately: 1) A source contributes from within approximately 1 optical depth. Beyond that distance its influence/contribution rapidly diminishes in an exponential fashion. 2) An optically thick system (in our case $\tau_{v}(L)$ being large) naturally approaches a BB spectrum. To see that simplify Eq. (1.14) by having no incident flux, $\mathrm{I}_{0}$, and let $\mathrm{I}_{\mathrm{BBv}}$ be constant in space and get:

$$
\mathrm{I}_{v}(\mathrm{~L})=\mathrm{I}_{\mathrm{BBv}}\left[1-\mathrm{e}^{-\tau v(\mathrm{~L})}\right]
$$

Let us now return to Eq. (1.10) and solve it in the so-called "diffusion approximation". We restrict ourselves to a planar geometry where quantities vary 
with $\mathrm{x}$. Thus a ray moving along in some arbitrary direction " $\mathrm{s}$ " that makes an angle $\theta$ with the $x$ axis (and define $\mu=\cos (\theta)$ so $d s=d x / \mu$ ), makes Eq. (1.10) become:

$$
\mu d I_{v} / d x=\kappa^{\prime}\left(I_{B B v}-I_{v}\right)
$$

We assume that $I_{v}$ deviates slightly from the isotropic $I_{B B v}: I_{v}=I_{B B v}+\mu \psi$

Plug that into Eq. (1.16) and obtain immediately:

$$
I_{v}=I_{B B v}-\mu\left(1 / \kappa^{\prime}\right) d I_{B B v} / d x
$$

Note that the second term is smaller than the first by $1 / \tau$. We can now calculate the net flux through an area perpendicular to the $\mathrm{x}$-axis:

$$
\mathrm{F}_{v}=\int_{-1}^{1} \mu \mathrm{I}_{v} 2 \pi \mathrm{d} \mu=-\frac{4 \pi}{3 \kappa_{v}^{\prime}} \frac{\mathrm{dI}_{v \mathrm{BB}}}{\mathrm{dx}}=-\frac{c}{3 \kappa_{v}^{\prime}} \frac{\mathrm{dU}_{v \mathrm{BB}}}{\mathrm{dx}}
$$

We can now integrate over $v$, and define a Rosseland ( $v$ averaged) $\mathrm{mfp}\left(1 / \mathrm{\kappa}^{\prime}\right)$, which is weighted in that average by $\mathrm{dU}_{\mathrm{BBv}} / \mathrm{dT}$ and get:

$$
\mathrm{F}=-\frac{\mathrm{c} \lambda_{\mathrm{R}}}{3} \frac{\mathrm{d}\left(\mathrm{aT} \mathrm{T}^{4}\right)}{\mathrm{dx}}=-\frac{4 \lambda_{\mathrm{R}}}{3} \frac{\mathrm{d}\left(\sigma \mathrm{T}^{4}\right)}{\mathrm{dx}}=-\frac{4}{3} \frac{\mathrm{d}\left(\sigma \mathrm{T}^{4}\right)}{\mathrm{d} \tau}
$$

This is a principal result that we will (eventually) use in the equation that couples the radiation to the matter. It tells us that radiant heat diffuses in a way entirely expected from diffusion theory- namely that the flux is a diffusion coefficient times a gradient of an energy density, and that the diffusion coefficient is a free streaming velocity times a mfp divided by 3. The final form for F of Eq. (1.19) tells us an equivalent message in a useful form- namely that the diffusive flux is the free-streaming flux reduced by the number of mfps (the optical depth) of the system.

Before proceeding to some applications we prepare the way with one more exercise in formalism. We integrate Eq. (1.17) over $v$, and define $\phi=\sigma \mathrm{T}^{4}$, and get:

$$
\mathrm{I}=(\phi / \pi)+(3 \mu / 4 \pi) \mathrm{F}=(\phi / \pi)-(\mu \lambda / \pi) \mathrm{d} \phi / \mathrm{dx}
$$

This form will now be very useful in exploring the diffusion picture in a regime where in principle it does not belong- namely near a boundary. Thus we encounter what are known as Milne boundary conditions.

Consider a source flux $\phi_{\mathrm{D}}$ going from left to right, as it encounters matter that fills space from $x>0$, and is heated to a depth $x_{F}$, and whose surface $T$ at $x=0$ is given by $\left(\phi_{\mathrm{B}} / \sigma\right)^{1 / 4}\left(\right.$ namely $\left.\mathrm{T}_{\mathrm{B}}\right)$. The source flux to the right at $\mathrm{x}=0$ is given by: 


$$
\overrightarrow{\mathrm{F}}_{\mathrm{R}}=\int_{0}^{1} 2 \pi \mu \mathrm{Id} \mu=\frac{2 \pi \phi_{\mathrm{D}}}{\pi} \int_{0}^{1} \mu \mathrm{d} \mu=\phi_{\mathrm{D}}
$$

and the flux, to the left at $x=0$, from the matter re-radiating is given by:

$$
\widehat{\mathrm{F}}_{\mathrm{L}}=\int_{0}^{-1} 2 \pi \mu \mathrm{Id} \mu=\frac{2 \pi \phi_{\mathrm{B}}}{\pi} \int_{0}^{-1} \mu \mathrm{d} \mu-2 \pi \frac{\lambda \frac{\mathrm{d} \phi}{\mathrm{dx}}}{\pi} \int_{0}^{-1} \mu^{2} \mathrm{~d} \mu=\phi_{\mathrm{B}}+\frac{2}{3} \lambda \frac{\mathrm{d} \phi}{\mathrm{dx}}
$$

so the net flux at $x=0$ is given by:

$$
\overrightarrow{\mathrm{F}}_{\mathrm{Net}}=\overrightarrow{\mathrm{F}}_{\mathrm{R}}-\overleftarrow{\mathrm{F}}_{\mathrm{L}}=\phi_{\mathrm{D}}-\phi_{\mathrm{B}}-\frac{2}{3} \lambda \frac{\mathrm{d} \phi}{\mathrm{dx}}
$$

This net flux must be carried inward into the material via the diffusive flux that we have already calculated in Eq. (1.19) namely $F=-(4 / 3) \lambda d \phi / d x$. Equating those 2 expressions for flux gives us:

$$
\phi_{\mathrm{D}}-\phi_{\mathrm{B}}=-\frac{2}{3} \lambda \frac{\mathrm{d} \phi}{\mathrm{dx}}, \Rightarrow \phi_{\mathrm{D}}=\phi_{\mathrm{B}}\left(1+\frac{\mathrm{F}}{2 \phi}\right)
$$

For a linear profile of $\phi(x)=\phi_{B}\left\{1-\left(x / x_{F}\right)\right\}$, Eq. (1.21) tells us that $\left.\phi_{D}=\phi_{B}\left\{1+(2 / 3) \lambda / x_{F}\right\}\right\}$, can which be thought of in the following way: that $\phi_{D}$ is a value higher than $\phi_{B}$ as if the linear profile if $\phi(x)$ was extended backward into the $x<0$ regime by an amount $2 / 3) \lambda$.

The same kinds of considerations can be applied to the surface of a star. Consider a half space $x<0$ filled with matter and whose surface temperature at $x=0$ is $T_{S}$. Then the flux to the right from the star is given by:

$$
\overrightarrow{\mathrm{F}}_{\mathrm{R}}=\int_{0}^{1} 2 \pi \mu \mathrm{Id} \mu=\frac{2 \pi \phi_{\mathrm{S}}^{1}}{\pi} \int_{0}^{1} \mu \mathrm{d} \mu-2 \pi \frac{\lambda \frac{\mathrm{d} \phi}{\mathrm{dx}}}{\pi} \int_{0}^{1} \mu^{2} \mathrm{~d} \mu=\phi_{\mathrm{s}}-\frac{2}{3} \lambda \frac{\mathrm{d} \phi}{\mathrm{dx}}
$$

and the flux to the left onto the star from the of vacuum of space is given by:

$$
\overline{\mathrm{F}}_{\mathrm{L}}=\int_{0}^{-1} 2 \pi \mu \mathrm{Id} \mu=0
$$

thus the net flux from the star is:

$$
\overrightarrow{\mathrm{F}}_{\mathrm{Net}}=\overrightarrow{\mathrm{F}}_{\mathrm{R}}-\widehat{\mathrm{F}}_{\mathrm{L}}=\phi_{\mathrm{S}}-\frac{2}{3} \lambda \frac{\mathrm{d} \phi}{\mathrm{dx}}
$$

But this net flux at $x=0$ must be supplied by the diffusive flux from within the star, which as usual is $\mathrm{F}=-(4 / 3) \lambda \mathrm{d} \phi / \mathrm{dx}$. Equating the 2 expressions for flux then gives:

$$
\phi_{\mathrm{S}}=-\frac{2}{3} \lambda \frac{\mathrm{d} \phi}{\mathrm{dx}}=\frac{\mathrm{F}}{2}, \Rightarrow \mathrm{F}_{\mathrm{Net}}=2 \phi_{\mathrm{S}}=2 \sigma \mathrm{T}_{\mathrm{S}}^{4}=\sigma \mathrm{T}_{\text {Brite }}^{4}
$$


This can be thought of again for a linear profile of $\phi(x)=\phi_{s}\{1-[x /(2 \lambda / 3)]\}$, as if the surface that "truly" is emitting, is at a distance $x=-2 / 3 \lambda$ within the star where $\mathrm{T}_{\text {Brite }}=\mathrm{T}(\mathrm{x}=0-(2 / 3) \lambda)=2^{1 / 4} \mathrm{~T}_{\mathrm{S}}$. This result is consistent with our "lesson (1)" from above, namely that emission comes from within about $1 \mathrm{mfp}$.

With all of this formalism now established, we are finally in a position to apply the results from this section to a variety of ICF relevant situations. We begin by finding a highly relevant case where Eq. (1.19) plays a crucial role.

\section{Solving the Diffusion Equation}

The first application we seek is the most relevant to the ICF indirect drive problem. We consider a hohlraum illuminated by a laser of energy $E_{L}$. It enters the hohlraum (usually made of a high $\mathrm{Z}$ material such as $\mathrm{Au}$ ) and is absorbed along the inner walls where it is aimed. The hot plasma that ensues is a copious source of $x-$ rays. We parameterize this process by a conversion efficiency $\eta_{\mathrm{CE}}$. Thus we assume that $\eta_{\mathrm{CE}} \mathrm{E}_{\mathrm{L}}$ worth of $\mathrm{x}$-rays now floods the hohlraum and uniformly bathes the wall areas of all that it sees. Some of the $\mathrm{x}$-rays are absorbed by the capsule (the goal of the exercise after all!) and some unfortunately leave the hohlraum through the laser entrance holes (LEH) necessary to get the laser into the hohlraum in the first place. Since the capsule ablator is normally of low $\mathrm{Z}$ material that does not re-radiate back much, it, like the LEH (which is the ultimate in energy sinks- a vacuum) absorb all the flux $\sigma \mathrm{T}^{4}$ that impinges on it. Thus we know immediately how to calculate those 2 energy loss channels: $\sigma T^{4}$ times the area of the capsule and LEH respectively, integrated over time.

Our major challenge is to calculate the wall loss, since it does re-radiate energy to a significant degree and moreover, the majority of area in the problem is the hohlraum wall. It is made of a high $\mathrm{Z}$ matter which when heated by the flux of incident $\mathrm{x}$-radiation will re-radiate much of it back. Typically, as we shall see, the re-radiation factor is not enough to disqualify the walls as the "chief energy loser"the preponderance of wall area still make the wall the principal sink of energy in ICF hohlraums. Since the incident $x$-rays are absorbed by remaining bound 
electrons of the multiply ionized ions of $\mathrm{Au}$, and then are re-radiated in a random direction as the electron finds a lower energy level to return to, we have a typical random walk / diffusive process. Collisions also keep the levels at near MB distributions (a situation called Local Thermodynamic Equilibrium (LTE)). This near total thermal equilibrium, near isotropic radiation field situation is precisely the conditions under which we derived the diffusive flux results from the previous section.

Thus our goal here is clear- to calculate the energy absorbed by a high $\mathrm{Z}$ wall subject to an external flux of x-rays. The time rate of change in internal energy (per unit volume) would be equal to the divergence of the diffusive flux. If the flux were divergence free then as much energy that entered a volume element of matter would then exit it and we'd expect no net change in the internal energy. For our system there are 2 fields of energy to consider: matter and radiation. The energy density of matter we write as $\rho \mathrm{e}_{\mathrm{th}}$, where e is the specific energy, and we know what it is for radiation: $\mathrm{aT}^{4}$. It turns out that the radiation energy density is quite negligible for the ICF problem. For the diffusive flux there are 2 contributions. We know what is for radiation: $F=-(4 / 3) \lambda d \phi / d x$. For matter, the electron heat flux is similarly given by a Dd(energy density $) d x=\left(v_{\mathrm{e}} \lambda_{\mathrm{e}} / 3\right) \mathrm{d}\left(\rho \mathrm{e}_{\mathrm{th}}\right) / \mathrm{dx}$. It turns out that for above about $20 \mathrm{eV}$ and density $1 \mathrm{~g} / \mathrm{cc}$ the radiative flux dominates. Thus our energy equation should read:

$$
\frac{\partial}{\partial t}\left(\rho e_{t h}\right)=\frac{\partial}{\partial x}\left(\frac{c \lambda_{R}}{3} \frac{\partial}{\partial x}\left(a T^{4}\right)\right)
$$

This is a complicated non-linear PDE. Before proceeding with any formal solutions, we can see how far simple dimensional analysis can take us. We write down Eq. (1.23) dimensionally:

$$
\frac{\rho e}{t} \sim \frac{1}{x} \lambda \frac{c a T^{4}}{x} \sim \frac{1}{x} \frac{1}{\kappa \rho} \frac{\sigma T^{4}}{x}
$$

(Note a slight change in nomenclature- we follow from here on out the convention that opacity $\kappa$ is defined as $1 / \rho \lambda$ [whereas in Eq. (1.9) it was simply $1 / \lambda]$. It is still true though that we always correct the absorption for the stimulated emission, so that it is net absorption). We can recast this in terms of the "Marshak front areal density" $\mathrm{m}_{\mathrm{F}}$ : 


$$
m_{F}^{2} \equiv\left(\rho X_{F}\right)^{2} \sim \frac{\sigma T^{4} \cdot t}{\kappa \cdot e}
$$

What we mean conceptually by this procedure is as follows: A non-linear heat wave of radiation (called a Marshak wave after R. E. Marshak (1958)) progresses diffusively through the material. Picture, at a given time, a flat-topped $\mathrm{T}(\mathrm{x})$ profile that eventually takes a sharp nose-dive to zero at a front position $\mathrm{x}_{\mathrm{F}}$. We will soon see that a $\mathrm{T}(\mathrm{x})=\mathrm{T}_{0}\left\{1-\left[\mathrm{x} / \mathrm{x}_{\mathrm{F}}(\mathrm{t})\right]\right\}^{1 / 4}$ is a good approximation to the actual solution. Since $T$ is nearly flat and therefore at its surface value for most of $x$, the dimensional analysis works particularly well in giving us a feel for the time and $\mathrm{T}$ dependence of the front position $x_{F}$ or $m_{F}$. To complete this analysis we need to know the $T, \rho$ dependencies of $\kappa$ and e. For Au we find that

$$
\kappa=\kappa_{0} \rho^{0.2} / \mathrm{T}^{1.5} \text { and } \mathrm{e}=\mathrm{e}_{0} \mathrm{~T}^{1.6} / \rho^{0.14}
$$

We note the remarkable near cancellation of dependencies of the product ке, which appears in the denominator of $\mathrm{m}_{\mathrm{F}}$ in Eq. (1.25). Why is this? Well the basic reason is that the opacity $\kappa$ depends on the number of bound electrons left in the partially ionized high $\mathrm{Z}$ ion, whereas the internal energy e depends on the oppositethe number of free electrons liberated from that high $\mathrm{Z}$ ion. (Note that e scales as $[(Z+1) / A] T)$. We can be somewhat more precise. The $T$ dependence of e can be understood as follows. We expect an ion to be ionized to the degree that its ionization potential $I_{p}$ is of order the thermal temperature $T$ (electron collisional ionization is the dominant process). But in a Bohr-like atom, $I_{p}$ scales a $Z^{2}$. Thus expect $Z$ to scale as $T^{1 / 2}$ and e as $T^{3 / 2}$ which it very nearly does! The opacity is quite complicated, but certainly it should decrease with $\mathrm{T}$ because its number of bound electrons is decreasing as $\mathrm{T}$ increases. For the density dependence we note that in a Saha equilibrium the higher the overall density, the lower the ionization state- its as if the free states are more full and prevent bound electrons from occupying themit's as if high density "pushes the electrons back into the atom". Thus the higher the $\rho$ the lower the $Z$ so e will scale as $(1 / \rho)$ to a small power. Since lower $Z$ means more bound electrons, the opacity $\kappa$ will increase as $\rho$ to a small power. In any event Eqs. (1.25) and (1.26) lead to:

$$
m_{F} \sim \frac{T^{1.95} t^{1 / 2}}{\sqrt{\kappa_{o} \cdot e_{o}} \rho^{0.03}}
$$


With such a tiny $\rho$ dependence you may think our task is done. But what we are really after is the energy (per unit area) in the wall which dimensionally is $\mathrm{em}_{\mathrm{F}}$ and e has some small but non-negligible density dependence. So our task in this dimensional analysis exercise is not complete until we find an expression for the density in terms of $\mathrm{T}$ and $\mathrm{t}$.

One way to do this is to assume we are deep into the supersonic regime- namely the heat wave progresses so fast through the material it hardly has time to move and thus the density is constant in space and time and at its initial value $\rho_{0}$. This will occur, at a given $\mathrm{T}$ and $\mathrm{t}$, for very low densities (imagine a high $\mathrm{Z} \mathrm{Au}$ foam). To see why, recast Eq. (1.27) in terms of $\mathrm{x}_{\mathrm{F}}=\mathrm{m}_{\mathrm{F}} / \rho$ and then differentiate with respect to time to get a velocity of the heat front. At a given $\mathrm{T} \& \mathrm{t}$ that speed will scale as $\rho^{-1.03}$. The sound speed $c_{\mathrm{s}}$ scales as $\mathrm{e}^{1 / 2}$ and thus as $\mathrm{e}_{0}{ }^{1 / 2} \mathrm{~T}^{0.8} \rho^{-0.078}$, a very weak $\rho$ dependence. Thus we can ensure that the heat front speed exceeds the sound speed for low $\rho$. Putting $\rho=$ constant $=\rho_{0}$ into Eq. (1.27) and into e gives us a scaling for energy loss per unit area in the supersonic regime:

$$
E / A \sim(\rho e) X_{F} \sim e m_{F} \sim \sqrt{\frac{e_{o}}{\kappa_{o}}} \frac{T^{3.55} t^{1 / 2}}{\rho_{o}^{0.17}}
$$

The exact opposite extreme is the highly subsonic regime, in which the heat front progresses very slowly through the solid density high $\mathrm{Z}$ material. What ends up happening is an isothermal rarefaction wave progresses through the heated material, significantly decompressing it and inducing much hydrodynamic motion in this "blow-off" plasma.

A simplified (and certainly dimensionally correct) way to proceed (Rosen (1979)) is to find an "average" density in this blow-off by reasoning that after some time $t$, an amount of mass (per unit area), $m_{F}(T, t, \rho)$, has been heated (reached by the heat front), and it expands into the vacuum at the sound speed $c_{S}(T, t, \rho)$, so simply set $\rho=\mathrm{m}_{\mathrm{F}} / \mathrm{c}_{\mathrm{S}}$ t. This accomplishes what we sought- a way to relate $\rho$ (implicitly) to $\mathrm{T} \& \mathrm{t}$. But since all $\rho$ dependencies of $\mathrm{m}_{\mathrm{F}}$ and $\mathrm{c}_{\mathrm{S}}$ are power laws it is straightforward to solve for $\rho$ explicitly in terms of $\mathrm{T} \& \mathrm{t}$, and then plug back in to solve for $\mathrm{m}_{\mathrm{F}}$ and then for $\mathrm{E} / \mathrm{A}$. Before we do that, we note a particular piece of luck here. Since $\mathrm{m}$ is formally a running integral of $\rho$ over $\mathrm{x}$ (it's basically a Lagrangian 
coordinate), then our simplified dimensional equation $\rho=\mathrm{m}_{\mathrm{F}} / \mathrm{c}_{\mathrm{S}}$ t is really an integral equation for $\rho$, whose solution is exactly the isothermal rarefaction! So proceeding with the substitutions we eventually get:

$$
\mathrm{m}_{\mathrm{F}} \sim \frac{\mathrm{T}^{1.91} \mathrm{t}^{.52}}{\left(\kappa_{0} \mathrm{e}_{0}\right)^{0.48}} \Rightarrow \frac{\mathrm{E}}{\mathrm{A}} \sim \mathrm{em}_{\mathrm{F}} \sim \frac{\mathrm{e}_{0}^{0.7}}{\kappa_{\mathrm{o}}^{0.4}} \mathrm{~T}^{3.34} \mathrm{t}^{\cdot 6}
$$

This completes our dimensional analysis. The hard work of determining the exact coefficients is what we briefly outline next.

The full set of hydrodynamic equations, in Lagrangian format $(\mathrm{m}, \operatorname{not} \mathrm{x})$ is:

$$
\text { (mass) } \frac{\partial \mathrm{V}}{\partial \mathrm{t}}=\frac{\partial \mathrm{u}}{\partial \mathrm{m}}, \text { (momentum) } \frac{\partial \mathrm{u}}{\partial \mathrm{t}}=-\frac{\partial \mathrm{P}}{\partial \mathrm{m}}, \text { energy) } \frac{\partial \mathrm{e}}{\partial \mathrm{t}}+\mathrm{P} \frac{\partial \mathrm{V}}{\partial \mathrm{t}}=\frac{4}{3} \frac{\partial}{\partial \mathrm{m}} \frac{1}{\kappa} \frac{\partial \sigma \mathrm{T}^{4}}{\partial \mathrm{m}}
$$

Here $V=1 / \rho$. We supplement these equations with equations of state a la Eq. (1.26), along with $\mathrm{P}=\mathrm{re} / \mathrm{V}$ where we find $\mathrm{r}=0.25$ to be reasonably accurate. For the record, we complete Eq. (1.26) by presenting the coefficients: $\kappa_{0}=7200 . \mathrm{g} / \mathrm{cm}^{2}$ and $\mathrm{e}_{0}=3.4$ MJ / g. In Hammer \& Rosen (H\&R) (2003) we solve (1.30) by means of a perturbation technique with a small parameter $\varepsilon=1.6 /(4+1.5)=0.29$ the numbers being the power law $\mathrm{T}$ dependencies of $\mathrm{e}, \mathrm{aT}^{4}, \& \kappa$ respectively. We do so for a particular, self-similar assumption on the $T(x, t)$ behavior, namely $T=T_{B} t^{k} f\left(m / m_{F}(t)\right)$ We find to zero order, a $\mathrm{T}$ spatial profile $\mathrm{f}=\left(1-\left(\mathrm{m} / \mathrm{m}_{\mathrm{F}}(\mathrm{t})\right)^{1 / 4}\right.$ where $\mathrm{m}_{\mathrm{F}}(\mathrm{t})=\mathrm{m}_{\mathrm{F}} \mathrm{t}^{(1+4 \mathrm{k}) / 2}$. The $\rho$ and $\mathrm{u}$ profiles are, to the same zero order, those of an isothermal rarefaction. The first order solutions differ from all these by quantities of order $\varepsilon$. In H\&R we verify energy conservation, integral $\mathrm{E}(\mathrm{x}, \mathrm{t}) \mathrm{dx}=$ integral $\mathrm{F}(\mathrm{x}=0, \mathrm{t}) \mathrm{dt}$ through order $\varepsilon^{2}$, where $\mathrm{E}$ includes internal and kinetic energy, and $\mathrm{F}$ is the absorbed flux. In addition, in H\&R we solve the simpler supersonic equation for arbitrary $T_{B}(t)$.

We quote here the results for 2 useful choices of $\mathrm{k}: 0 \& 0.18$. The scaling of $\mathrm{m}$ \& E/A are precisely those of Eq. (1.29) as they must be! The coefficients are $\mathrm{m}_{\mathrm{F} 0}=$ $(9.9,7.4) 10^{-4} \mathrm{~g} / \mathrm{cm}^{2}$ respectively, and $\mathrm{E} / \mathrm{A}=(0.58,0.39) \mathrm{hJ} / \mathrm{mm}^{2}$ respectively. The absorbed flux is given by $F=F_{0} T^{3.34} t^{-0.41}$ with coefficients $F_{0}=(0.34,0.46) \mathrm{hJ} / \mathrm{ns} / \mathrm{mm}^{2}$. Note that E/A is simply the time integral of $\mathrm{F}$. Also be aware that for the $\mathrm{k}=0.18$ case you must remember to put the time dependence of $T=T_{0} t^{0.18}$ into all of these equations. Thus for example the $\mathrm{E} / \mathrm{A}($ for $\mathrm{k}=0.18)=0.39 \mathrm{~T}_{0}{ }^{3.34} \mathrm{t}^{1.2} \mathrm{hJ} / \mathrm{mm}^{2}$. 
It seems unfair to present all of these results without giving the reader a sense of how they come about. So we now proceed to show an illustrative example of an exact solution for the supersonic (constant $\rho$ ) case. Thus we start with

$$
\frac{\partial}{\partial \mathrm{t}} \mathrm{T}^{\beta}=\mathrm{C} \frac{\partial^{2}}{\partial \mathrm{x}^{2}} \mathrm{~T}^{4+\alpha}, \text { with } C=\frac{16}{(4+\alpha)} \frac{\mathrm{g} \sigma}{3 \mathrm{f} \rho^{2-\mu+\lambda}}
$$

where here we generalize the $T, \rho$ power law behavior of $\kappa$ and e and write them as $\kappa=(1 / \mathrm{g}) \mathrm{T}^{-\alpha} \rho^{\lambda}$ and $\mathrm{e}=\mathrm{fT}^{\beta} \rho^{-\mu}$. Thus for Au (see Eq. (1.26) $\alpha=1.5, \beta=1.6, \lambda=0.2, \mu=0.14$, and the coefficients are $(1 / \mathrm{g})=7200 . \mathrm{g} / \mathrm{cm}^{2}$ and $\mathrm{f}=3.4 \mathrm{MJ} / \mathrm{g}$. In the early 1950's Louis Henyey found 2 exact solutions to Eq. (1.31). This work remains unpublished so in $H \& R$ we devoted an appendix to present it. The simpler of the 2 solutions is what we now present. Henyey took a power law time dependence $t^{p}$ for the boundary temperature with $p=1 /(4+\alpha-\beta)$, which for $A u$ is approximately $1 / 4$. He then finds:

$$
\mathrm{T}(\mathrm{x}, \mathrm{t})=\mathrm{T}_{\mathrm{B}} \mathrm{t}^{1 /(4+\alpha-\beta)}\left(1-\frac{\mathrm{x}}{\mathrm{x}_{\mathrm{F} 0} \mathrm{t}}\right)^{1 /(4+\alpha-\beta)} \text { with } \quad \mathrm{x}_{\mathrm{F} 0}^{2}=\mathrm{C} \frac{4+\alpha}{4+\alpha-\beta} \mathrm{T}_{\mathrm{B}}^{4+\alpha-\beta}
$$

The reader should verify this by plugging Eq. (1.32) into (1.31) and experience the fun of terms miraculously canceling and verifying that indeed Eq. (1.32) solves Eq. (1.31) exactly. Note that this solution looks very much like the advertised zero order solution of H\&R, namely a spatial profile that looks like $\left\{1-\left(\mathrm{x} / \mathrm{x}_{\mathrm{F}}(\mathrm{t})\right)\right\}^{1 / 4}$ and with the $\mathrm{T}=\mathrm{T}_{\mathrm{B}} \mathrm{t}^{\mathrm{k}=1 / 4}$, we get the front advancement behavior $\mathrm{x}_{\mathrm{F}}(\mathrm{t})=\mathrm{x}_{\mathrm{F} 0} \mathrm{t}^{(1+4 \mathrm{k}) / 2}\left(=\mathrm{x}_{\mathrm{F} 0} \mathrm{t}^{1}\right.$ here). Since $\mathrm{p}=1 / 4$ is a small number it is easy to accept that other (non-exact solution) cases that might be of interest such as $k=0$, will simply be of order $\varepsilon$ different from this solution. Using this exact solution we can derive some interesting quantities, especially the very useful one of wall reflectivity, albedo.

\section{IV.Solving for the Albedo}

With an exact solution in hand let us proceed to calculate the fundamental quantity of diffusion processes, the optical depth $\tau$. To simplify the notation set $\mathrm{y}=\mathrm{x} / \mathrm{x}_{\mathrm{F} 0} \mathrm{t}$. Then, in this supersonic (constant $\rho$ ) regime, $\lambda=\left(\kappa_{0} \rho_{0}{ }^{-1} \mathrm{~T}^{\alpha}\right.$, thus

$$
\tau=\int \frac{\mathrm{dx}}{\lambda}=\kappa_{0} \rho_{0} \int \frac{\mathrm{dx}}{\mathrm{T}^{\alpha}}=\left(\frac{\kappa_{0} \rho_{0} \mathrm{x}_{\mathrm{F}} \mathrm{t}}{\mathrm{T}_{\mathrm{B}}^{\alpha} \mathrm{t}^{\alpha \mathrm{p}}} \int_{0}^{y} \frac{\mathrm{dy}}{(1-\mathrm{y})^{\alpha /(4+\alpha-\beta)}}\right.
$$




$$
=\left(\frac{\kappa_{0} \rho_{0} \mathrm{x}_{\mathrm{F}} \mathrm{t}}{\mathrm{T}_{\mathrm{B}}^{\alpha} \mathrm{t}^{\alpha \mathrm{p}}}\right)\left(\frac{4+\alpha-\beta}{4-\beta}\right)\left(1-(1-\mathrm{y})^{\frac{4-\beta}{4+\alpha-\beta}}\right) \equiv \tau_{\mathrm{F}}\left(1-(1-\mathrm{y})^{\frac{4-\beta}{4+\alpha-\beta}}\right)
$$

Note that $\tau_{\mathrm{F}}$ represents the total number of mfps within the Marshak wave. Note that it increases with time as $\mathrm{t} / \mathrm{t}^{1.5 / 4}=\mathrm{t}^{0.6}$. We can invert Eq. (1.33) to get:

$$
(1-y)=\left(1-\frac{\tau}{\tau_{\mathrm{F}}}\right)^{\frac{4+\alpha-\beta}{4-\beta}} \text { and thus } \mathrm{T}(\tau)=\mathrm{T}_{\mathrm{B}}(1-\mathrm{y})^{\frac{1}{4+\alpha-\beta}}=\mathrm{T}_{\mathrm{B}}\left(1-\frac{\tau}{\tau_{\mathrm{F}}}\right)^{\frac{1}{4-\beta}}
$$

In Figure (1) we show the $\mathrm{T}(\mathrm{y})$ and $\mathrm{T}(\tau)$ profiles for a situation $\varepsilon$ different than our exact solution, namely a "NIF-like" $250 \mathrm{eV}, 7.7 \mathrm{~ns}$ "flat top" $\left(\mathrm{T}_{B}=\mathrm{T}_{0} \mathrm{t}^{0}\right)$ drive. It shows shapes very much like those predicted by Eq. (1.34). For the record, the H\&R solutions for that case are $T(y)=T_{B}\left(1-y{ }^{1.2}\right)^{0.25}(1-0.05 y)$ and $T(\tau)=T_{B}\left(1-\left[\tau / \tau_{F}\right]\right)^{0.42}(1-$ $0.1\left[\tau / \tau_{\mathrm{F}}\right]$ ) which indeed are only $\varepsilon$ different than Eq. (1.34). Also for the record, putting in the constants we find for that case that $\tau_{\mathrm{F}}=8.4 \mathrm{~T}_{0}{ }^{0.65} \mathrm{t}^{0.4}$ (with $\mathrm{T}$ in heV and $t$ in $n s)=34$ by the end of the 7.7 ns pulse.
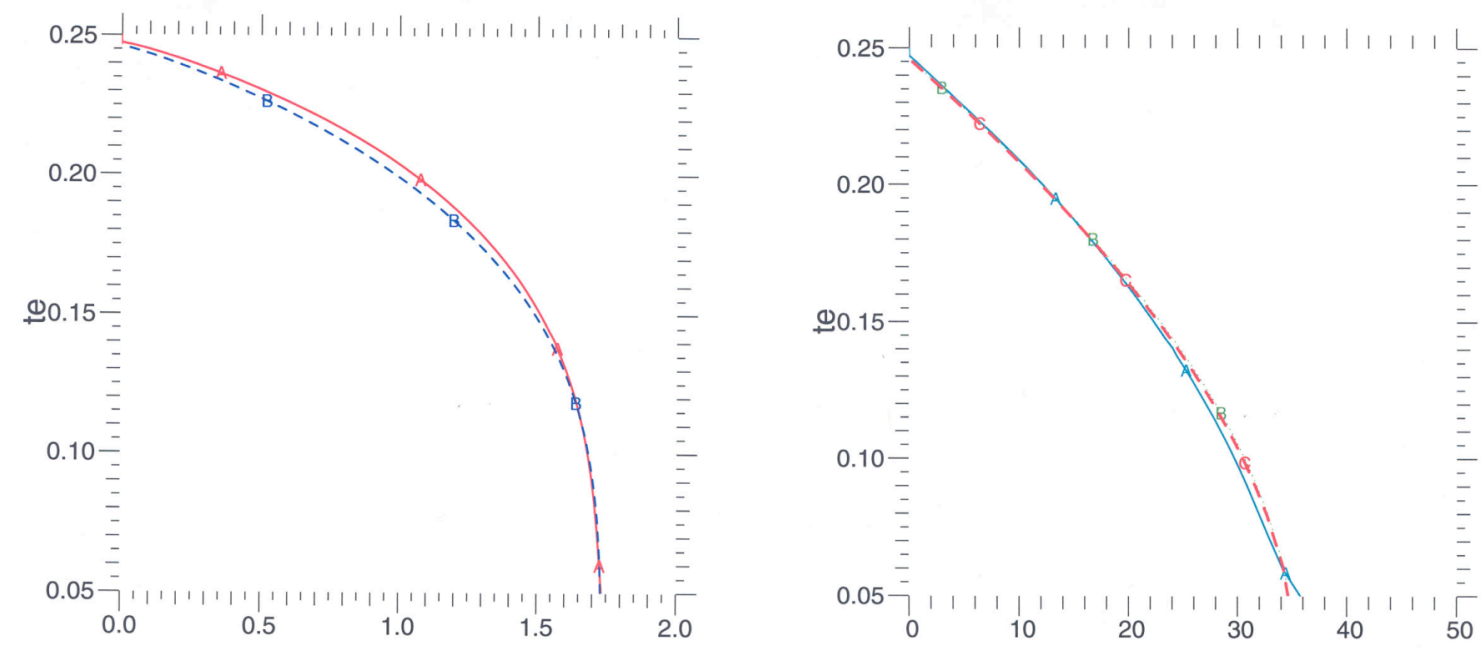

Figure 1 a) $\mathrm{T}(\mathrm{m})$ profile $\&$ b) $\mathrm{T}(\tau)$ profile for a $250 \mathrm{eV} 7.7 \mathrm{~ns}$ drive. (Dotted lines are analytic theory and solid lines are numerical solutions).

With the temperature profile now written in its fundamental form, in terms of $\tau$, we can now straight away solve the radiation transport equation to find how much this "source profile" $\mathrm{I}=\sigma \mathrm{T}(\tau)^{4} / \pi$ will radiate back into the vacuum. Since we know most of the radiation will come from near the first mfp, we can simplify the calculation by expanding $\mathrm{T}(\tau)^{4}$ near the surface $\left(\tau / \tau_{\mathrm{F}}\right.$ small), and we now proceed to integrate the emission $I(\mu)$ all along a ray that makes an angle $\theta$ with the $x$ axis with $\cos \theta=\mu$ : 


$$
\begin{array}{r}
\mathrm{I}_{\mu}=\left(\frac{\sigma \mathrm{T}_{\mathrm{B}}^{4} \mathrm{t}^{4 \mathrm{p}}}{\pi}\right) \int_{0}^{\tau_{\mathrm{F}} / \mu}\left(1-\frac{4}{(4-\beta)} \frac{\tau}{\tau_{\mathrm{F}}}\right) e^{-\tau / \mu} \mathrm{d} \tau / \mu \\
\approx\left(\frac{\sigma \mathrm{T}_{\mathrm{B}}^{4} \mathrm{t}^{4 \mathrm{p}}}{\pi}\right)\left[1-\left(\frac{4}{4-\beta}\right) \frac{\mu}{\tau_{\mathrm{F}}}\right]+O\left(e^{-\tau_{F}}\right)
\end{array}
$$

(Note that because all of our quantities vary with $x$, we have defined $\tau$ here as $\tau(x)$, not as the simpler $\tau(\mathrm{s})$ as before, hence the $\tau / \mu$ terms in Eq. (1.35) vs. Eq. (1.14)). Then the total reemission can be found by integrating over all angles of emission:

$$
\mathrm{I}_{\mathrm{Tot}}=\int_{0}^{1} 2 \pi \mu \mathrm{I}_{\mu} \mathrm{d} \mu=\left(\sigma \mathrm{T}_{\mathrm{B}}^{4} \mathrm{t}^{4 \mathrm{p}}\right)\left[1-\left(\frac{4}{4-\beta}\right) \frac{(2 / 3)}{\tau_{\mathrm{F}}}\right]
$$

Another immediate outgrowth of Eq. (1.34) is our ability to calculate the absorbed flux (through the $x=\tau=0$ front surface) immediately from the last form for $F$ of Eq. (1.19)

$$
\mathrm{F}=-\frac{4}{3} \frac{\mathrm{d}\left(\sigma \mathrm{T}^{4}\right)}{\mathrm{d} \tau}=\left(\frac{4}{3}\right)\left(\sigma \mathrm{T}_{\mathrm{B}}^{4} \mathrm{t}^{4 \mathrm{p}}\right)\left(\frac{4}{4-\beta}\right) \frac{1}{\tau_{\mathrm{F}}}
$$

When considering Eqs. (1.36) and (1.37) we are struck with a paradox. We would expect the sum of the re-radiated flux (Eq. (1.36) plus the absorbed flux (Eq. (1.37)) to add up to the incident flux $\sigma \mathrm{T}_{\mathrm{B}}{ }^{4} \mathrm{t}^{4 \mathrm{p}}$. But they do not! The $1 / \tau_{\mathrm{F}}$ terms have coefficients $-2 / 3$ and $+4 / 3$. What went wrong?

The answer lies in the Milne boundary conditions. We have defined all of our Marshak wave solutions in terms of $T_{B}$ which is the matter boundary temperature. This makes no statement whatsoever as to what is the required external drive temperature $T_{D}$ needed to achieve that boundary temperature. That is what the Milne boundary conditions tell us. In fact Eq. (1.21) tells us that $\mathrm{T}_{\mathrm{D}}{ }^{4}=\mathrm{T}_{\mathrm{B}}{ }^{4}\left(1+\left(\mathrm{F} / 2 \mathrm{~T}^{4}\right)\right)$. If we employ that relationship into our energy accounting all turns out well, as we now demonstrate.

Using Eq. (1.37) we can re-write Eq. (1.36) as re-emitted flux $=\phi_{\text {в }}(1-(\mathrm{F} / 2 \phi))$, and Eq. (1.37) as absorbed flux $\mathrm{F}=\phi_{\mathrm{B}}(\mathrm{F} / \phi)$, and from the Milne boundary condition, incident flux $=\phi_{\text {в }}(1+(\mathrm{F} / 2 \phi))$. Now it is self-evident that energy is conserved and that incident flux $=$ absorbed + re-emitted flux. Now with energy conservation safely within our grasp we can consider albedo $\alpha$ as the ratio of re-emitted flux to incident flux that conserves energy: $\alpha=1-(\mathrm{F} / \phi)$. Since $\mathrm{F} / \phi$ is proportional to $1 / \tau_{\mathrm{F}}$, and $\tau_{\mathrm{F}}$ grows in time, we expect both the albedo and the Milne correction to 
approach 1 as time increases. This is shown rather clearly in Fig. (2). The conditions of Fig. (2) are a $\mathrm{Ta}_{2} \mathrm{O}_{5} 40 \mathrm{mg} / \mathrm{cc}$ foam driven by a flat $\mathrm{T}$ of $185 \mathrm{eV}$.
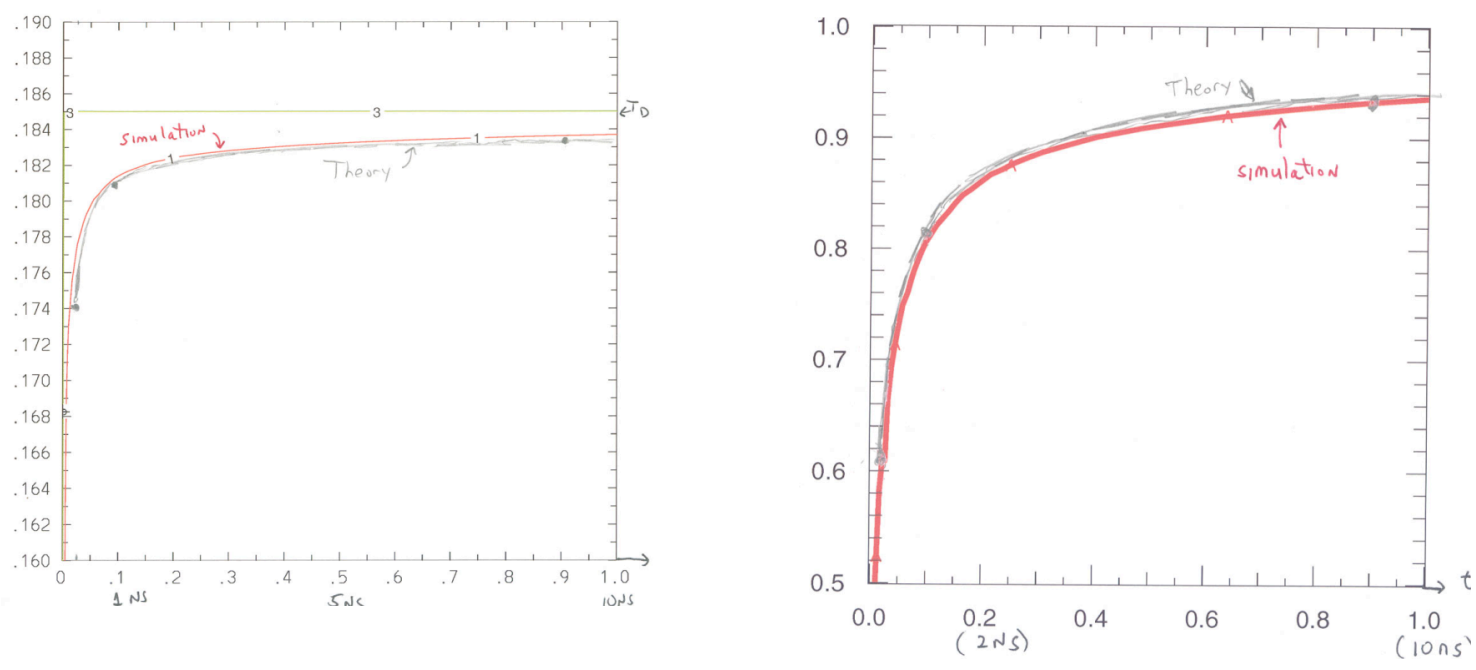

Figure 2 a) Milne correction \& b) albedo as they both approach 1 with time.

Before proceeding, let's make sure we haven't gotten lost in all of the math. Let us ask what we might have guessed the formula for emitted flux to be. Based on our "lesson number 1" we know that emission comes from about the first mfp. To fine-tune it a bit from our Milne boundary condition calculations it seemed like 2/3 of a mfp was the more precise answer. Thus we would expect re-emission to be $\mathrm{T}^{4}$ but $\mathrm{T}$ is evaluated at $2 / 3$ of a mfp into the Marshak profile. Thus using Eq. (1.34):

$$
\mathrm{T}^{4}(\tau=2 / 3)=\mathrm{T}_{\mathrm{B}}^{4}(\mathrm{t})\left[1-\frac{(2 / 3)}{\tau_{\mathrm{F}}}\right]^{\left(\frac{4}{4-\beta}\right)} \approx \mathrm{T}_{\mathrm{B}}^{4}(t)\left[1-\left(\frac{4}{4-\beta}\right) \frac{(2 / 3)}{\tau_{\mathrm{F}}}\right]
$$

which is precisely the re-emission formula we calculated in Eq. (1.36). One more "reality check" is in order. Eq. (1.35) tells us to expect the re-emission to be angle dependent so experimentalists must be aware of this as they choose angles of detection. The physics of this may well be called "Marshak limb brightening". Basically, if you look normal to a wall, the emission coming from $1 \mathrm{mfp}$ comes from deep in the wall, and thus at a low $\mathrm{T}$ within the Marshak wave $\mathrm{T}(\mathrm{x})$ profile. If you look at a shallow angle to the wall, then along that ray you will reach $1 \mathrm{mfp}$ in, yet you will hardly be different than $\mathrm{x}=0$, so you will be getting emission from a high $\mathrm{T}$ 
very near the surface of the $T(x)$ Marshak wave profile. Fig. (3) illustrates this principle for the same conditions as Fig. (2).
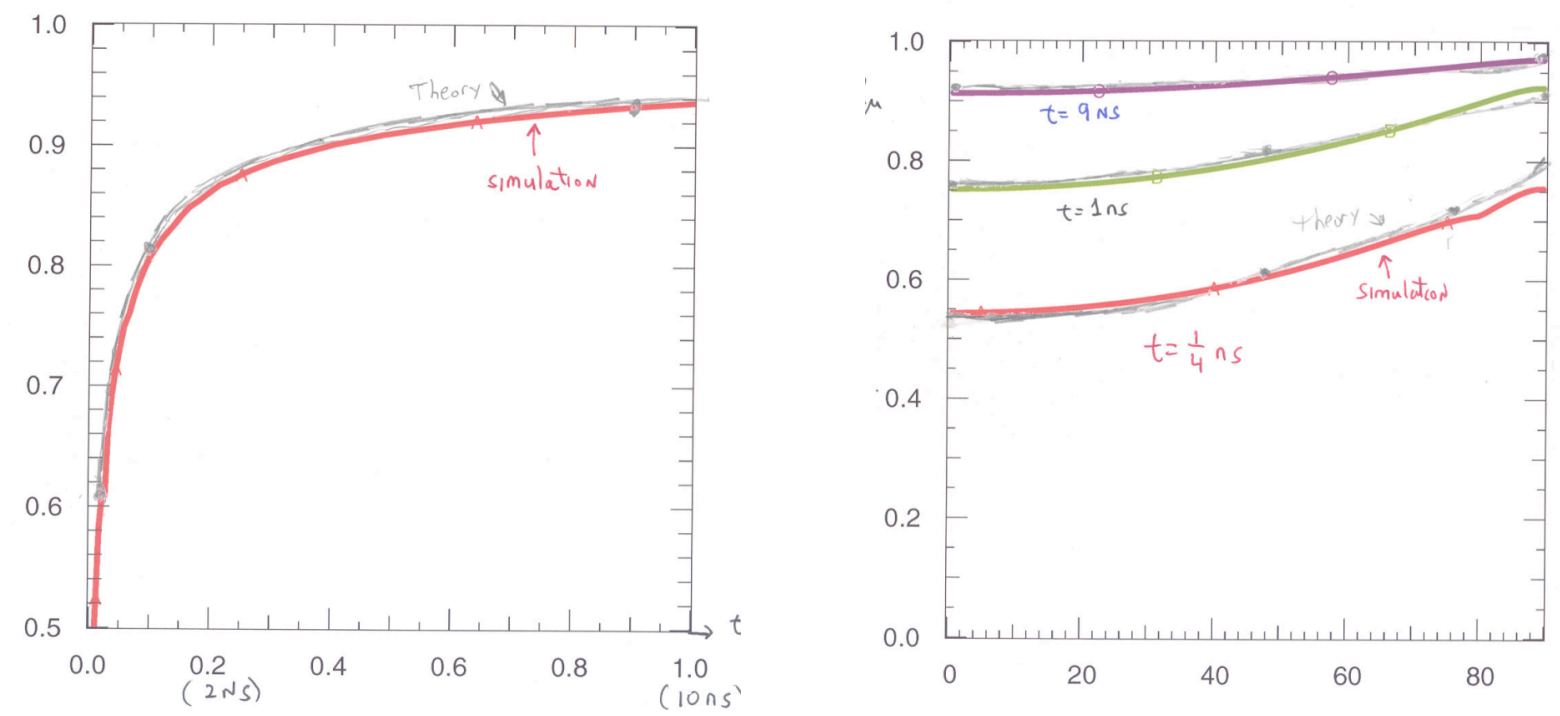

Figure 3 a) Angle averaged albedo vs. time \& b) Albedo vs. angle for 3 different times. $\operatorname{Cos}\left(48^{\circ}\right)=2 / 3$ represents the angle averaged result

As a final application of this albedo section we compare predictions based on our analytic work with full simulations and data. Jones et al (2004) measured the albedo of a large hohlraum wall that was fed by a smaller hotter hohlraum. The flux out of the exit hole of the small hot hohlraum was measured and that is the incident flux impinging onto the large hohlraum's wall. The flux re-emitted by that larger hohlraum was also measured and the ratio was interpreted as an albedo. There are 4 data points. The first 2 are $\mathrm{Au}$ at $70 \mathrm{eV}$ and at $100 \mathrm{eV}$ (both at $1.5 \mathrm{~ns}$ ) with experiment and simulation giving albedoes of 0.53 and 0.63 respectively. Using $\alpha=$ 1- $(\mathrm{F} / \phi)$ with $\mathrm{F}$ given by $\mathrm{H} \& \mathrm{R}$ for the hohlraum relevant $\mathrm{k}=0.18$ case (more about that in the next section), namely $\mathrm{F} / \phi=0.46 / \mathrm{T}^{0.7} \mathrm{t}^{0.53}$ ( $\mathrm{T}$ in $\mathrm{heV}, \mathrm{t}$ in $\mathrm{ns}$ ) we would predict albedoes of $0.53,0.63$ respectively as well! For a $\mathrm{Au}_{0.2} \mathrm{Dy}_{0.2} \mathrm{U}_{0.6}$ "cocktail" (more about those in Lecture 2) at $100 \mathrm{eV}$ and $1.5 \mathrm{~ns}$ we predict a slight decrease in opacity and an albedo of 0.62 , which again is what the experiment measures and the simulations predict. For $\mathrm{U}_{0.86} \mathrm{Nb}_{0.14}$ the experiment shows an albedo of 0.65 but the simulations predict 0.53 . We do not understand that result to date. Our model matches the simulation (that's the best it can do in any event!) with a prediction of 
0.54. Thus our simple formulae do a very good job of matching complex simulations (which in turn, often match complex experimental data).

\section{Solving for the hohlraum temperature}

We are now very well positioned for our principal application- for a given hohlraum geometry and incident laser pulse, predict the hohlraum temperature. We will then compare that with data so let us first describe the measurement techniques (Kaufman (1994)). We typically obtain $\mathrm{T}$ by two different methods. Through a hole in the can (sometimes on the side, sometimes a view through the LEH) we measure the x-ray flux via a dozen or so roughly $100 \mathrm{eV}$ wide ("Dante") channels that span an energy from $50 \mathrm{eV}$ to $5 \mathrm{keV}$ with most of the energy in the 100-1000 eV range where most Planckian spectra lie for Ts of order 100-300 eV. A Planckian is fit to the resultant spectrum, and it usually matches in both brightness (intensity) and color (shape). Examples of Dante spectra are given in Figure (4).

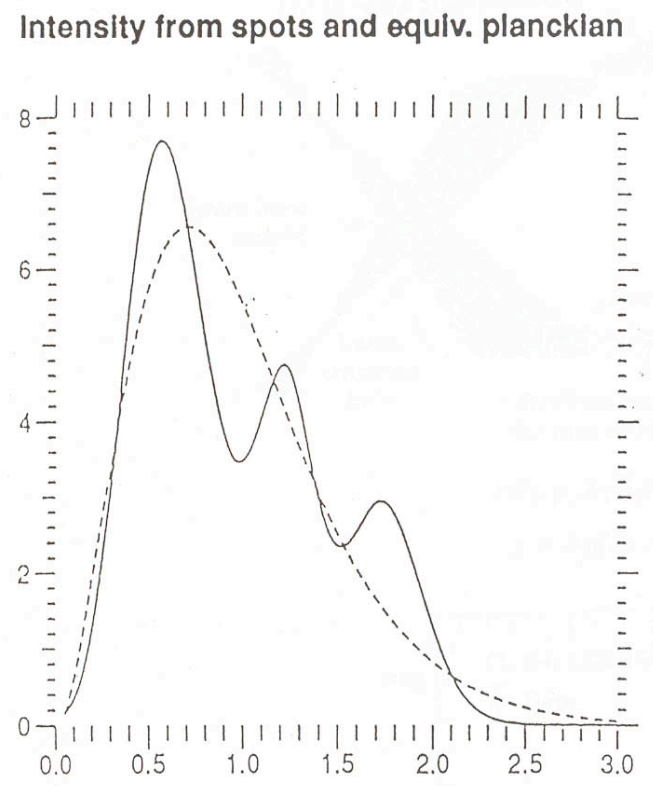

laser illuminated spot
Intensity from spots and equiv. plancklan

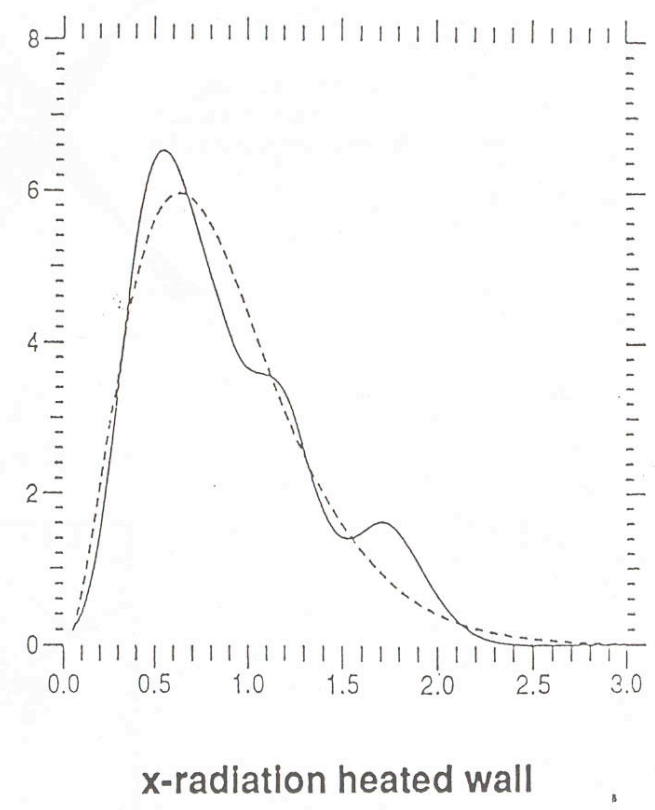

Figure 4 Observed spectra from a) a laser illuminated spot b) an un-illuminated spot on the hohlraum wall. 
Since these channels have some decent time resolution we can get $\mathrm{T}(\mathrm{t})$ information as well. The second method involves an $\mathrm{Al}$ "witness plate" on the side of the hohlraum. Radiative flux absorbed sub-sonically in the Al launches a shock. When the shock breaks out the back of the $\mathrm{Al}$ it lights up in the visible range, and a streaked optical signal detector records the time history of the shock breakout. For a stepped witness plate we can derive a shock speed, hence a pressure, hence a drive T. For a wedge shaped witness plate we can even derive a more sophisticated pulse shaped $\mathrm{T}(\mathrm{t})$. In general the 2 methods match, as do the Lasnex (Zimmerman(1975)) simulations. See Fig. (5).
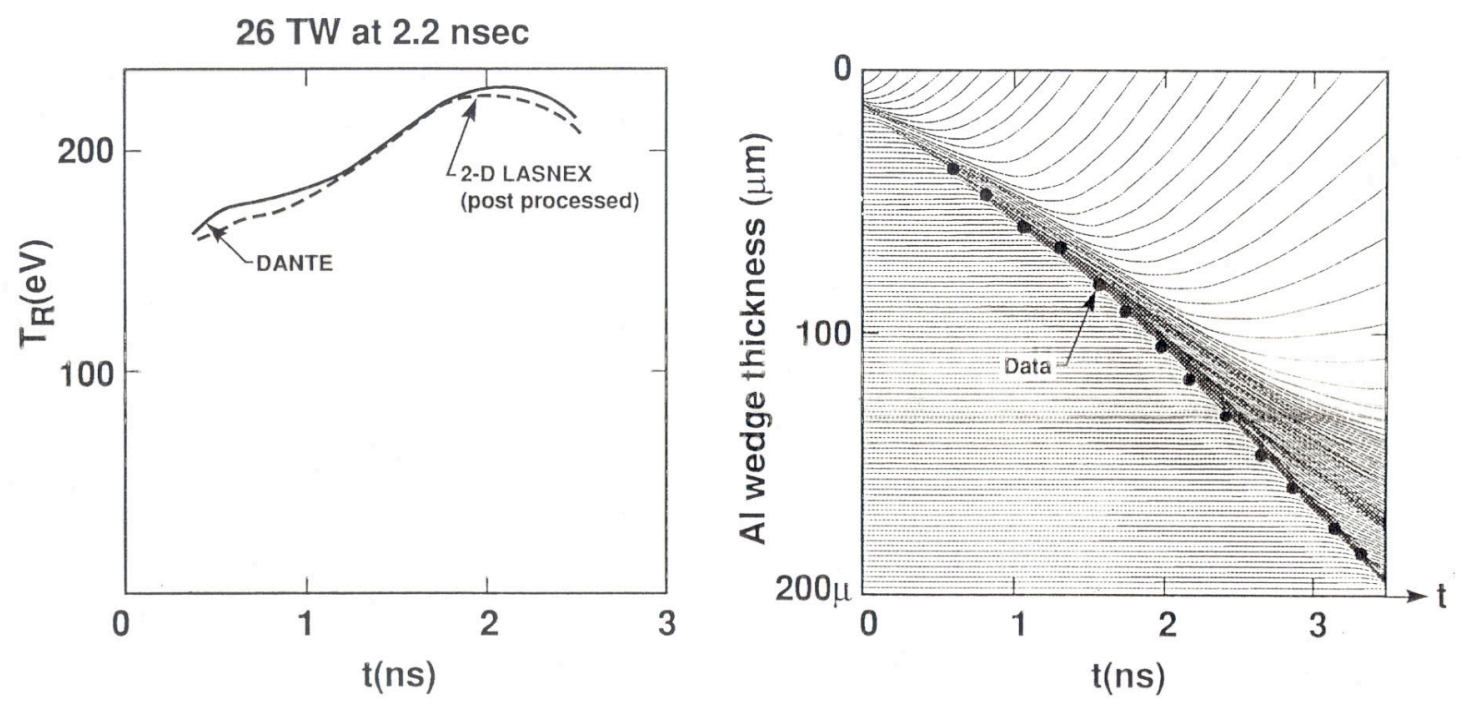

Pulse is $2.2 \mathrm{nsec}, 1: 3$ foot to main pulse power ratio

Figure 5 a) Time history of a shaped pulse from Dante and from Lasnex simulation.

b) Applying that Lasnex simulation to an Al wedge witness plate predicts the experimentally observed temporal history of the shock break out.

To calculate all of this analytically we adopt a simple "source=sink" model. The source is the laser energy $E_{\mathrm{L}}$ and as described above in the beginning of Section 3 , we assume it is converted to $x$-rays with some conversion efficiency, so that now $\eta_{\mathrm{CE}} \mathrm{E}_{\mathrm{L}}$ worth of $\mathrm{x}$-rays bathe the hohlraum walls. (If the absorption fraction is less than one, of course we should take that into account as well). We will set that 
source equal to the energy sinks, which for a very simple hohlraum (no capsule) is the wall loss (which we have calculated as E/ A of the previous 2 sections times the area of the walls, and the LEH loss which is the time integral of $\sigma \mathrm{T}^{4}$ times the area of the laser entrance holes. Before presenting those results, let us first introduce convenient "radiation hohlraum units" ("rhu") in which $\mathrm{T}$ is measured in hectovolts (hundreds of $\mathrm{eV}$ ), area in $\mathrm{mm}^{2}$, time in $\mathrm{ns}$, mass in gm and energy (a bit clumsily) in hectojoules. With these units, $\sigma=1$, (well, 1.03 to be exact) and normalized irradiance is $10^{13} \mathrm{~W} / \mathrm{cm}^{2}\left(=\mathrm{hJ} / \mathrm{mm}^{2} \mathrm{~ns}=10^{2} \mathrm{~J} / 10^{-2} \mathrm{~cm}^{2} 10^{-9} \mathrm{~s}\right)$ and similarly, normalized power is $10^{11} \mathrm{~W}\left(=\mathrm{hJ} / \mathrm{ns}=10^{2} \mathrm{~J} / 10^{-9} \mathrm{~s}\right)$. Thus a $100 \mathrm{eV}(=1 \mathrm{heV})$ hohlraum radiates out a hole with a flux of $\sigma T^{4}=1$ (in rhu) $=10^{13} \mathrm{~W} / \mathrm{cm}^{2}$.

As an example we take the following "scale 1" hohlraum illuminated on the Nova laser at LLNL in the 1990's. It was a gold cylinder of length $\mathrm{L}=2.5 \mathrm{~mm}$, and radius $\mathrm{R}=0.8 \mathrm{~mm}$, and on each end a disk sealed the cylinder. Each disk had a "50\% LEH" namely a laser entrance hole of radius $0.4 \mathrm{~mm}$. One immediately calculates the wall area $A_{W}=15.6 \mathrm{~mm}^{2}$ and $A_{L E H}=1 \mathrm{~mm}^{2}$. The source energy, a "flat top" laser power of $100-300 \mathrm{hJ} / \mathrm{ns}$ for a duration of $1 \mathrm{~ns}$. (=10-30 TW). Our simulations predict a conversion efficiency to x-rays of $\eta_{\mathrm{CE}}=0.7 \mathrm{t}_{\mathrm{ns}}^{0.2}$. The efficiency increases with time in part because the albedo behind the conversion layer builds up with time. This time behavior helps explain an important experimental observation, that $\mathrm{T}$ rises as $\mathrm{t}^{0.18}$, hence our interest as quoted above with the $\mathrm{k}=0.18$ case. We can understand that result in the following way. Equating the x-ray source, $\eta_{\mathrm{CE}} \mathrm{E}_{\mathrm{L}}=\eta_{\mathrm{CE}} \mathrm{P}_{\mathrm{L}} \mathrm{t}$ which scales as $\mathrm{t}^{1.2}$ to the principal $\mathrm{x}$-ray sink, the wall, $\mathrm{E}_{\mathrm{W}}$ which scales as $\mathrm{T}^{3.3} \mathrm{t}^{0.6}$ (Eq. (1.29)) we see that these two terms will balance iff $\mathrm{T}=$ $\mathrm{T}_{0} \mathrm{t}^{0.18}$ ! Conversely, if we wish to have a truly flat $\mathrm{T}=\mathrm{T}_{0} \mathrm{t}^{0}$, we need a $\mathrm{P}_{\mathrm{L}}(\mathrm{t})$ that "droops" in time.

Let us now proceed directly to the calculation. For the $30 \mathrm{TW}$ experiment, the source of $x$-rays (at $1 \mathrm{~ns}$ ) will be $0.7 \mathrm{P}_{\mathrm{L}} \mathrm{t}=(0.7)(300)(1)=210 \mathrm{hJ}$. The wall loss $\mathrm{E}_{\mathrm{W}}$ will be (using the $k=0.18$ results from $H \& R$ quoted in the discussion that followed Eq. (1.30) above) $0.39 \mathrm{~T}_{0}^{3.3} \mathrm{t}^{1.2} \mathrm{~A}_{\mathrm{W}}=0.39 \mathrm{~T}_{0}{ }^{3.3}$ (1) (15.6) $=6.24 \mathrm{~T}_{0}^{3.3}$ at $1 \mathrm{~ns}$. We must also calculate the LEH loss. The flux out the LEH will be $\mathrm{T}^{4} \mathrm{~A}_{\mathrm{LEH}}$ so we integrate $\mathrm{t}^{4(0.18)}$ in time and get $\mathrm{E}_{\mathrm{LEH}}=0.58 \mathrm{~T}^{4}$ at $1 \mathrm{~ns}$. Solving $210=6.24 \mathrm{~T}_{0}{ }^{3.3}+0.58 \mathrm{~T}^{4}$ results in a $\mathrm{T}=2.75$ with $176 \mathrm{hJ}$ of wall loss and $34 \mathrm{hJ}$ of LEH loss (justifying our claim that most 
of the loss is in the walls). The resulting prediction of $275 \mathrm{eV}$ matches data and simulations quite well. Repeating this calculation for say $10 \mathrm{TW}$ (70 replaces 210) yields a $\mathrm{T}=1.99$ again in agreement with data and simulation. The results for the entire database are shown in Figure (6), and shows that our simple model of source $=$ sink with sinks calculated by H\&R organize the database very nicely.

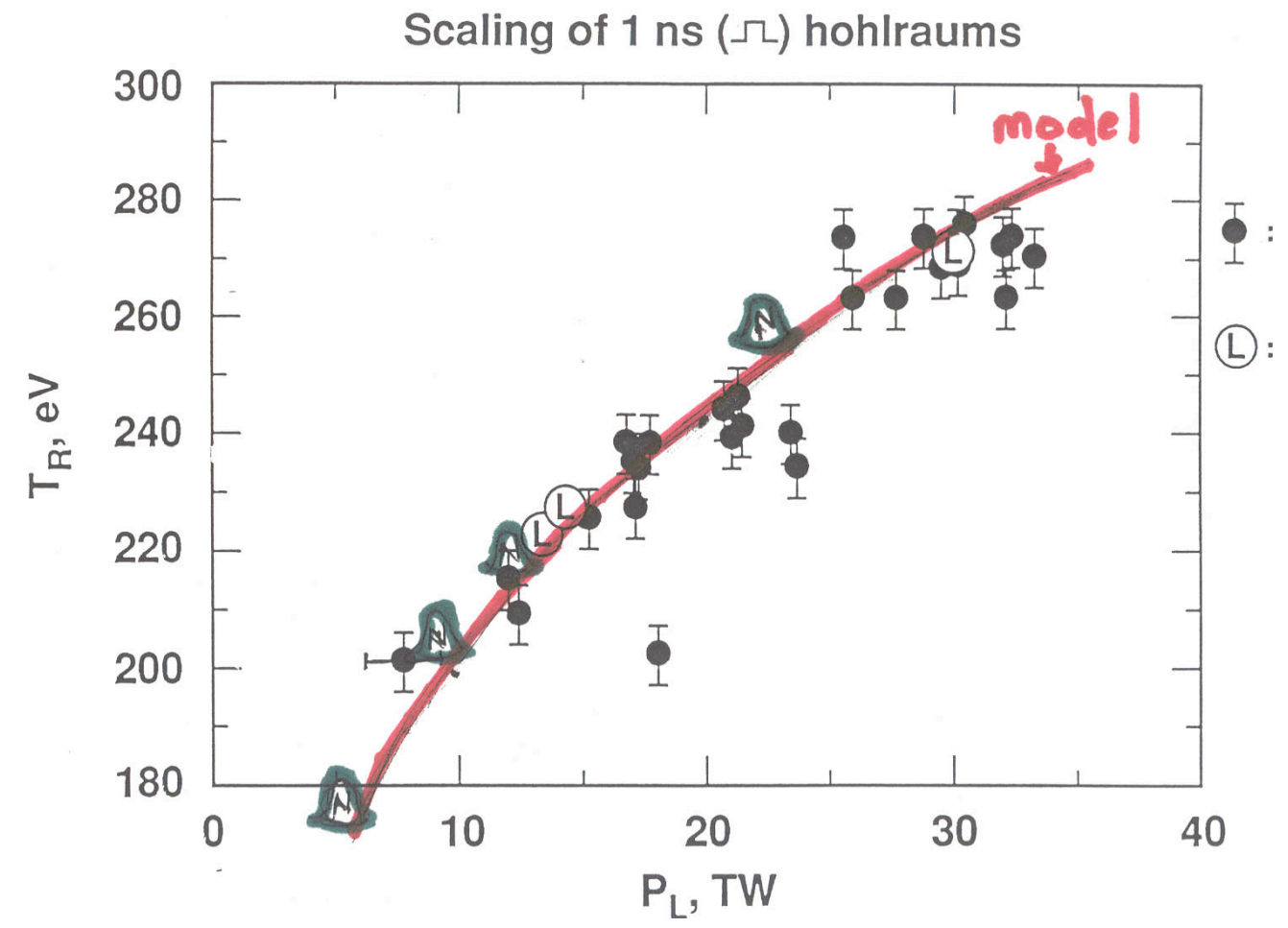

Figure 6 Our simple source $=$ sink model (line) organizes the 1 ns database very well. Solid points are from Nova. L means Lasnex 2-D simulation. $N$ means NIF early light experiments ( $2 \mathrm{~ns}$ pulses, with $\mathrm{T}$ here extracted from that data at $\mathrm{t}=1 \mathrm{~ns}$ ).

As a final application of what we have learned so far, consider scaling to bigger systems (say from Nova to NIF to a reactor scale driver). We seek to improve the coupling efficiency defined as energy absorbed by the capsule to incident laser energy. Since most of the energy is absorbed and turned into x-rays, and most of those are lost into the walls, this principally reduces to a question of the capsule loss divided by the wall loss. Now the capsule ablator is low $\mathrm{Z}$ so it just sucks up radiation energy and does not re-emit much, so it absorbs mostly like an LEH, so $E_{\text {cap }}$ scales roughly as $A_{\text {cap }} T^{4} t$. The wall loss $E_{W}$ we now know well, scales roughly as $0.5 A_{W} T^{3} t^{1 / 2}$. Thus the ratio, the coupling efficiency scales as $\left(A_{\text {cap }} / A_{W}\right) 2 . T t^{1 / 2}$. To 
improve the coupling which of these four factors can we change? The wall ratio is mostly fixed by geometric smoothing symmetry arguments. The value for the drive $\mathrm{T}$ is mostly fixed by hydrodynamic instability and laser plasma interaction physics. However as we increase the driver energy scale, the size scale will increase as will the time scale. So that is at least one natural way the coupling will certainly improve. An entire other route to improving the ratio, would be to change the number 2. Namely, are there wall loss mitigation tricks that we can test on present lasers that can be used on future laser driven hohlraums to improve the coupling efficiency? Answering that question is the subject of Lecture II. 


\section{Lecture II: Hohlraum optimization}

\section{Introduction to Lecture II:}

There is always, in practice, pressure on the scientists to keep a laser from operating safely away from its damage thresholds. That means using less laser energy. So we ask the question: Can the NIF reach ignition while using only $1 \mathrm{MJ}$ of energy rather than 2? To do so we would need to find ways to make hohlraum walls less lossy than the standard solid Au. In this lecture we discuss the state of research that has been trying to answer this question.

\section{Cocktails}

When we consider Eq. (1.29) we see that in order to lower the E/A of a wall loss, we need to lower e and to raise $\mathrm{k}$. Since e scales as $\mathrm{Z} / \mathrm{A}$ the higher the $\mathrm{Z}_{\mathrm{N}}$ (or A) the lower (at a given $T$ ) will be the ionization state $Z$ and hence e. Thus either replacing $\mathrm{Au}$ with $\mathrm{U}$ or at least mixing in higher $Z_{\mathrm{N}}$ elements into the wall will lower e. Moreover, if we do mix in higher $Z_{N}$ elements, at a given $T$, they will have different atomic levels and structures and their opacities, if $Z_{N}$ is chosen properly will be high at frequencies when Au is low. Overall this "cocktail" of materials can thus accomplish both things. $\mathrm{A}_{0.6} \mathrm{Dy}_{0.2} \mathrm{Au}_{0.2}$ cocktail is an example that at NIF like temperatures of about $300 \mathrm{eV}$ can, by Eq. (1.29) save nearly $20 \%$ in wall loss. We have chosen to test the concept via a baseline Au hohlraum at the Omega Laser at URLLE. A “scale 0.75 " cylinder ( $\mathrm{L}=2.06 \mathrm{~mm}, \mathrm{R}=0.6 \mathrm{~mm}$, with $66 \% \mathrm{LEH}(\mathrm{R}=0.4 \mathrm{~mm})$ so that $A_{W}=A_{\text {end caps }}+A_{\text {cyl wall }}=1.2+7.8=9.0 \mathrm{~mm}^{2}$ and $A_{L E H}=1 \mathrm{~mm}^{2}$. The incident flattop power was $20 \mathrm{TW}$ for 1ns. As in the discussion of Section V we use the $\mathrm{k}=0.18$ results of $\mathrm{H} \& \mathrm{R}$. We infer (from some experiments) about an $8 \%$ reflectivity, so with a $68 \%$ conversion efficiency we get as a source at $1 \mathrm{~ns} 101 \mathrm{hJ}$. We set that equal to the wall loss $3.5 \mathrm{~T}_{0}{ }^{3.3}$ and LEH loss $0.6 \mathrm{~T}_{0}{ }^{4}$, solve for $\mathrm{T}$ and get $\mathrm{T}=2.55$ with 76 hJ wall loss and $25 \mathrm{LEH}$ loss. The $255 \mathrm{eV}$ is very close to the observed temperature.

Early experiments with cocktails (Orzechowski (1996)) compared the burnthrough times $t_{b t}$ of $\mathrm{Au}$ foils (Rosen(1996)) placed across a hole in the side of a 260 
$\mathrm{eV}$ hohlraum, to those of AuGd cocktail foils. A delay in burn-through signal for the cocktail was clearly seen. By Eq. (1.28) we expect (again for a $k=0.18$ case) that $t_{b t}$ should scale as $\mathrm{mT}_{0}^{-2}(\mathrm{e} \kappa)^{1 / 2}$, so the higher $\kappa$ of the AuGd cocktail causes the delay. Since then we have tried to measure the rise in $\mathrm{T}$ for a full cocktail (vs. $\mathrm{Au}$ ) hohlraum at the same laser drive.

We fit our latest opacity/ EOS theory of $\mathrm{Au}$ as $\kappa=6544 \rho^{0.18} / \mathrm{T}^{1.43}\left(\mathrm{~cm}^{2} / \mathrm{g}\right)$ and $\mathrm{e}=3.33 \mathrm{~T}^{1.54} / \rho^{0.15}(\mathrm{MJ} / \mathrm{g})$, and of $\mathrm{U}_{0.6} \mathrm{Dy}_{0.2} \mathrm{Au}_{0.2}$ is $\kappa=5670 \rho^{0.10} / \mathrm{T}^{0.90}\left(\mathrm{~cm}^{2} / \mathrm{g}\right)$ and $\mathrm{e}=0.95 \mathrm{e}_{\mathrm{Au}}$. The cocktail has a "flatter", less sensitive $\mathrm{T}, \rho$ behavior because it averages over several elements. We also note that the opacity of cocktails does not exceed that of Au until past $130 \mathrm{eV}$. Using that input, $\mathrm{H} \& \mathrm{R}$ predicts for $\mathrm{k}=(0,0.18)$, for $\mathrm{Au}$ an $\mathrm{E} / \mathrm{A}=(0.598,0.398) \mathrm{T}^{3.3 \mathrm{t}^{0.6}}\left(\mathrm{hJ} / \mathrm{mm}^{2}\right)$ respectively and for the $\mathrm{U}_{0.6} \mathrm{Dy}_{0.2} \mathrm{Au}_{0.2}$ $\mathrm{E} / \mathrm{A}=(0.604,0.407) \mathrm{T}^{3.1} \mathrm{t}^{0.57}(\mathrm{hJ} / \mathrm{mm} 2)$ respectively. Thus, at $270 \mathrm{eV}$ and $1 \mathrm{~ns}$, the wall loss ratio (cocktail/ $\mathrm{Au})$ is $(0.84,0.85)$ respectively while a full multi-group simulation gives $(0.85,0.87)$, very close to $H \& R$ theory but differing mostly because the opacity is hard to fit with a single power law. All of these were for $T_{B}$ scaling as $t^{k}$. If we simply let $T_{D}$ scale as $t^{k}$ we have Milne conditions kicking in and the ratios are $(0.84,0.88)$ respectively. For $\mathrm{U}$ mixed with $6 \% \mathrm{Nb}$ by weight $(=14$ atom $\%)$ add $1 \%$ to all those ratios.

Another outgrowth of these scaling laws is to notice that the wall loss ratio scales as $\mathrm{T}^{-0.22} \mathrm{t}^{-0.05}$ for $\mathrm{k}=0.18$ and even for the "flat top " $\mathrm{k}=0$ case the wall loss ratio has a $\mathrm{t}^{-0.02}$ ratio. Thus to the degree that the Omega experiments are not either at the full NIF temperature of $300 \mathrm{eV}$, nor at the NIF pulse length of 3-4 ns, then the results from such experiments will be pessimistic in showing a wall loss ratio advantage of a cocktail hohlraum over Au than would a NIF ignition hohlraum. (The ratio for NIF is about 0.83 ). All of these time behaviors stem from the fact that early in time the lower $\mathrm{T}$ parts of the Marshak wave profile are relatively more important, and for low $\mathrm{T}$ the cocktail is actually worse than Au.

So let us redo the Omega hohlraum calculation for $\mathrm{T}$ with cocktail walls (actually shot with $\mathrm{U}_{0.86} \mathrm{Nb}_{0.14}$ ) and thus our $\mathrm{E} / \mathrm{A}$ wall loss is $0.416 \mathrm{~T}^{3.1}$ at $1 \mathrm{~ns} \mathrm{vs}$. $\mathrm{Au}$ $0.39 \mathrm{~T}^{3.3}$. The solution now to $101=3.7 \mathrm{~T}^{3.1}+0.6 \mathrm{~T}^{4}$ is $\mathrm{T}=2.62$ so we expect a $7 \mathrm{eV}$ hotter hohlraum than the $255 \mathrm{eV}$ Au hohlraum. Many shots were done with $\mathrm{Au}$ end plates and just a cylinder body of cocktail. Redoing that we must solve 101= 
$0.49 \mathrm{~T}^{3.3}+3.2 \mathrm{~T}^{3.1}+0.6 \mathrm{~T}^{4}$ we get 2.61 thus we expect a $6 \mathrm{eV}$ improvement for those type of cocktail hohlraums. However, until very recently there was barely any (less than $3 \mathrm{eV}$ ) difference between Au and cocktail hohlraums- what went wrong?

We formulated the following hypothesis-perhaps oxygen contaminated the cocktail walls in the process of making them. Since cocktail foils don't necessarily get leached from their substrates, cocktails hohlraums certainly do and the leaching process may be the key to the contamination. While Au does not bind to O, U \& Dy certainly do - they are in fact often used as $O$ getters! The trouble with $O$ in the cocktail is that they are fully ionized so contribute about twice the $\mathrm{Z}$ per unit weight than the high $\mathrm{Z}$ elements, and thus raise e by raising the specific heat.

For atomic numbers between 6 and 71 and for $T$ between $1 \& 3$ heV we find the following fits for the specific energy e. The ideal gas law would give $\mathrm{e}_{\mathrm{ig}}=15$ $[(\mathrm{Z}+1) / \mathrm{A}] \mathrm{T}_{\mathrm{heV}}$ in $\mathrm{MJ} / \mathrm{g}$. Here the ionization state $\mathrm{Z}$ is fit by $\mathrm{Z}=\left(\mathrm{Z}_{\mathrm{N}} / 71\right)^{0.6} 16 \mathrm{~T}_{\mathrm{heV}}{ }^{0.6} /$ $\rho_{\mathrm{g} / \mathrm{cc}}{ }^{0.14}$. There are non-ideal gas law contributions, principally from ionization energy, and we fit those by a multiplier "mult" $=2.5\left(\mathrm{Z}_{\mathrm{N}} / 71\right)^{0.1}$ Thus $\mathrm{e}=\left(\mathrm{e}_{\mathrm{ig}}\right)$ ("mult") which we can write as $\delta_{N} / A_{N}$. The reader can check that this gives a number reasonably close to the one we quoted for $\mathrm{Au}$ above. For a mixture of $\mathrm{j}$ elements we take the ratio e $=\Sigma \delta_{\mathrm{Nj}} / \Sigma \mathrm{A}_{\mathrm{Nj}}$. AuNdDy cocktail to be discussed shortly, we find $\mathrm{e}_{\mathrm{c}} / \mathrm{e}_{\mathrm{Au}}=1.06$ with no oxygen, 1.08 with $4 \% \mathrm{O}$, and 1.22 with $40 \% \mathrm{O}$.

A vivid example of the $\mathrm{O}$ problem came from a re-analysis of another burnthrough experiment, that of Olsen et. al. (2003). A AuNdDy cocktail foil burnthrough time $\left(t_{\text {b.t.c }}\right)$ was compared to that of an Au foil's in a $160 \mathrm{eV}$ hohlraum with a $\mathrm{T}$ that rose as $\mathrm{t}^{0.1}$. This would lead us to predict that (via the equivalent of Eq. (1.29)) the ratio of $(\kappa e)_{c} /(\kappa e)_{\text {Au }}$ would equal $\left.\left[(\rho \Delta x)_{A u} /(\rho \Delta x)_{c}\right]\right]^{2.08}$ times $\left[t_{\text {b.t.c }} / t_{\text {b.t.Au }}\right]^{1.5}$. Plugging in the data that equals $[1.9 / 1.5]^{2.08}$ times $[1.3 \mathrm{~ns} / 1.45 \mathrm{~ns}]^{1.5}=1.38$. Now theoretically the $(\kappa е)_{c} /(\kappa e)_{\text {Au }}$ ratio should 1.22 which disagrees with the data. But if there were an $\mathrm{O}$ for each Dy and for each $\mathrm{Nd}$, then theoretically the ratio is 1.36 quite close (and well within error bars) of the data. Thus due to this re-analysis, we "post-dicted" that the sample were fully oxygenated. We then had the target fabrication records examined and indeed that was precisely the case! Of course, now that we are aware of this issue, future targets can be carefully made without $\mathrm{O}$. 
Assuming the older hohlraum shots at Omega were also fully oxygenated (we'll never really know- the state of those hohlraums at shot time was not characterized) we can redo our source $=$ sink model once again but with a lossier wall loss due to the high e due to the oxygen. Now the E/A coefficient is 0.44 and, with Au end plates we solve $101=0.49 \mathrm{~T}^{3.3}+3.4 \mathrm{~T}^{3.1}+0.6 \mathrm{~T}^{4}$ and get $\mathrm{T}=2.575$, thus we in retrospect should have only expected a $2.5 \mathrm{eV}$ difference from the $255 \mathrm{eV}$ pure $\mathrm{Au}$ hohlraums, in rather close agreement with what was observed. The good news is that very recent shots in which great care has been taken to avoid oxygenation, has shown a $\Delta \mathrm{T}$ much closer to our original expectations for cocktails. Part of the way to prevent the oxygen from getting into the sample is to coat it with a thin layer of $\mathrm{Au}$. (roughly $0.2 \mathrm{~m}$ ). Even that layer lowers $\Delta \mathrm{T}$ somewhat, since we are replacing cocktail with $\mathrm{Au}$. However for the NIF, that same thickness of Au can still do the Oxygen prevention job, while being an utterly trivial fraction of the Marshak depth and hence not cause any worrisome detriment to the cocktail wall loss advantage. Thus with a few more confirmatory shots, we are well within grasp of proving the cocktail principle as an energy saver for NIF.

\section{VI.Foam-walled Hohlraums}

We now ask the following question: Can we save on driver energy by making hohlraum walls out of low density high $\mathrm{Z}$ foams, which have less hydrodynamic motion (namely less radiation heated and ablated material that streams back into the hohlraum interior as a low density isothermal blow-off) and hence, reduced net absorbed energy by the walls? We answer this question using our HR analytic theory, as well as by numerical simulations. To the degree that the "pure" HR theory diverges from the simulations we derive non-ideal non-self-similar corrections to the theory that bring it into agreement with the simulations (Rosen (2005)). We show that low-density high $\mathrm{Z}$ foams can indeed bring a savings of $20 \%$ in the required driver energy. Remarkably, this reduction is universalindependent of drive $\mathrm{T}$ and its pulse-duration $\mathrm{t}$. We derive an analytic expression for the optimal density (for any given $\mathrm{T}$ and $\mathrm{t}$ ) that will achieve this reduction factor and which agrees very well with numerical simulations. Such an approach might allow more routine operation of the National Ignition Facility (NIF) with laser 
energy further away from the optics damage threshold, and still provide the nominal energy (as originally designed with solid wall hohlraums) to the fuel capsule. Reduced hydrodynamic motion of the wall material may also reduce symmetry swings, as found for heavy ion beam targets.

For the sake of brevity and clarity we will restrict our study here to a drive that is constant in time for a duration t. Solving the supersonic Marshak wave, (Eq. (1.31) for $\mathrm{Au}$, our small parameter, $\varepsilon=0.291$ and the constant $\mathrm{C}$ is given by $4.08 \times 10^{-7}$ $/ \rho^{2.06} \mathrm{~cm}^{2} / \mathrm{ns}$. For those parameters we find $x_{F}^{2}=[(2+\varepsilon) /(1-\varepsilon)] C \mathrm{~T}^{4+\alpha-\beta} \mathrm{t}$, which, for our case gives $x_{F}(\mathrm{~cm})=0.0012 \mathrm{~T}^{1.95} \mathrm{t}^{.5} / \rho^{1.03}$. Our solutions for the energy per unit area, $\mathrm{E} / \mathrm{A}$, (in $\mathrm{MJ} / \mathrm{cm}^{2}$ ) absorbed by the gold wall is:

$$
\mathrm{E} / \mathrm{A}=0.0029 \mathrm{~T}^{3.55} \mathrm{t}^{5} / \rho^{0.17} \quad \text { (for the pure supersonic regime). (2.1) }
$$

For the sub-sonic solution, we repeat what we presented above: For $\mathrm{k}=0$, we found $\mathrm{m}_{\mathrm{F}}(\mathrm{t})=\mathrm{m}_{0} \mathrm{~T}_{\mathrm{S}}(\mathrm{t})^{1.91} \mathrm{t}^{0.52}$ with $\mathrm{m}_{0}=9.9010^{-4} \mathrm{~g} / \mathrm{cm}^{2}$ and (in MJ $/ \mathrm{cm}^{2}$ )

$\mathrm{E} / \mathrm{A}=0.0058 \mathrm{~T}^{3.35} \mathrm{t}^{.59} \quad$ (for the pure subsonic regime).

Comparing Eqs. (2.1) and (2.2) we see (for a typical drive of $\mathrm{T}=2.5 \mathrm{heV}$ and duration $\mathrm{t}=2 \mathrm{~ns}$ ) that for densities in the neighborhood of $0.3 \mathrm{gm} / \mathrm{cc}$ there is clearly less wall loss for the supersonic case. Lowering densities further decreases opacity and increases specific heat, both in the undesirable direction of more loss to internal energy (as per Eq. (1.28)). Raising densities would be desirable as that would lower wall losses even further, but unfortunately it would take us into the subsonic regime. The sound speed, $\mathrm{C}_{\mathrm{S}}$, at $250 \mathrm{eV}$ in gold is about $60 \mu \mathrm{m} / \mathrm{ns}$, which (using the expression for $x_{F}$ that precedes Eq. (2.1)) exceeds the supersonic heat front velocity, $\mathrm{d} x_{F} / \mathrm{dt}$, at $2 \mathrm{~ns}$ when $\rho_{0}$ is about $0.5 \mathrm{gm} / \mathrm{cc}$.

In Fig. (7) we plot $E$ / A vs. initial $\rho_{0}$ of the wall from Eq. (2.1) (for $\mathrm{T}=2.5$ and $t=$ 2.) and we plot the subsonic ("infinite density") result of Eq. (2.2) as well. We also plot the numerical simulation results. Note that Eq. (2.1) closely matches the full physics numerical simulations, deep in the supersonic regime (at very low $\rho_{0}$ ) when little hydrodynamic motion is expected. When hydrodynamic motion is artificially turned off in numerical simulations (not shown here), Eq. (2.1) closely matches those artificial simulations for all densities. 


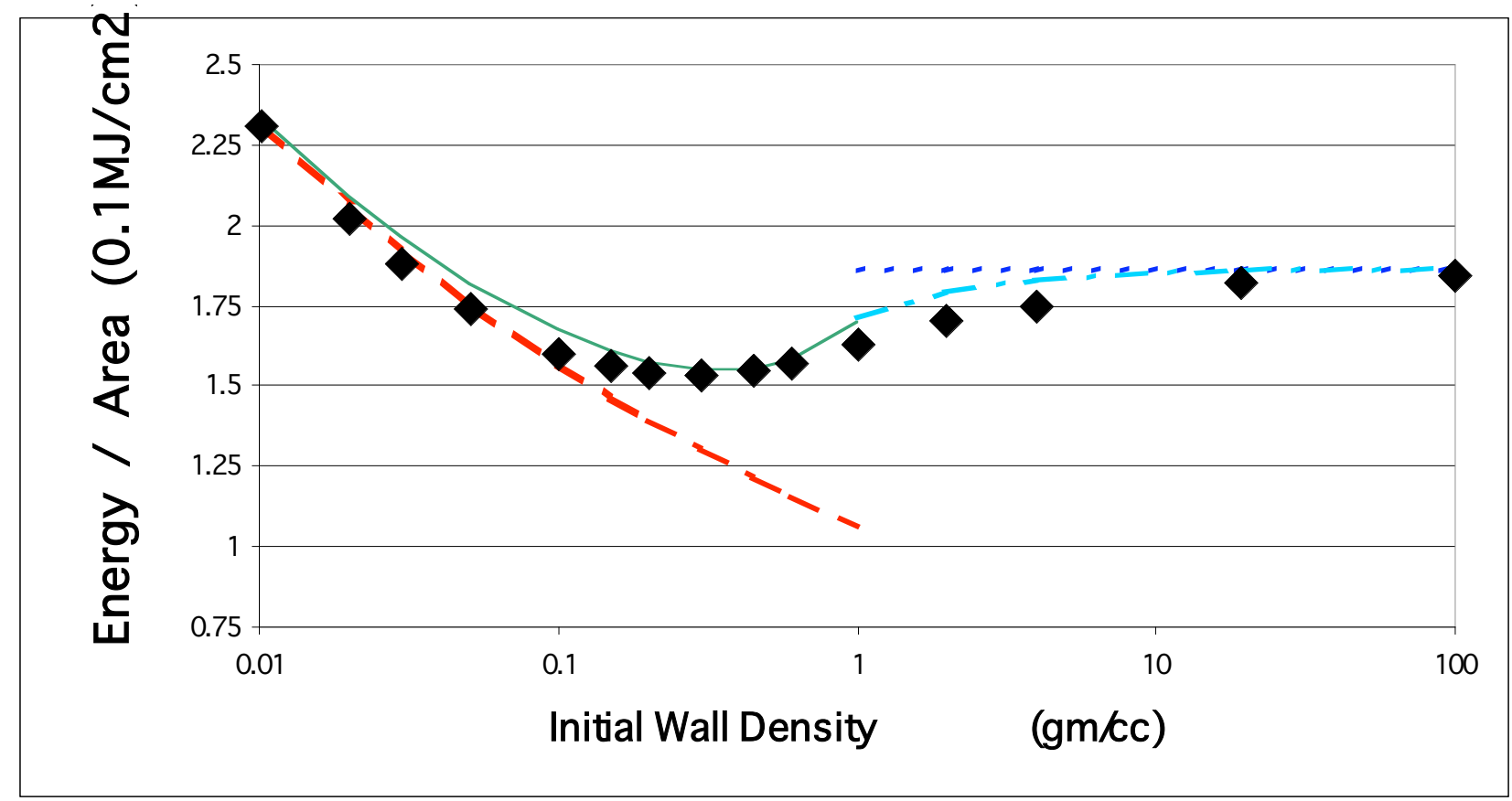

Figure 7 Wall loss $\left(0.1 \mathrm{MJ} / \mathrm{cm}^{2}\right)$ vs. initial wall density $(\mathrm{g} / \mathrm{cc})$. Diamond points are simulation results. Dashed line: Eq. (2.1). Dotted line: Eq (2.2). Solid line: Eq. (2.3). Dot-Dashed line: Eq. (2.4). Drive Conditions: $\mathrm{T}=250 \mathrm{eV}$; Duration $\mathrm{t}=2 \mathrm{~ns}$

To account for the divergence of the full physics simulations from our self similar solutions we reason as follows: In the supersonic regime but at higher $\rho_{0}$, rarefactions do in fact begin to eat into that portion of the heated wall nearest the drive boundary and hydro motion ensues. Consider an isothermal rarefaction wave propagating leftward (at speed $\mathrm{C}_{\mathrm{S}}$ ) into an $\mathrm{x}<0$ half space of temperature $\mathrm{T}$ and original constant density $\rho_{0}$, which results in low density material blowing out, rightward. If we define $z(x, t)=1+\left(x / C_{S} t\right)$, then the density profile is given by $\rho(x, t)$ $=\rho_{0} \exp (-z)$ and the velocity profile is given by $\mathrm{U}(\mathrm{x}, \mathrm{t})=\mathrm{z} \mathrm{C}_{\mathrm{S}}$. Within the rarefaction the kinetic energy per unit area at any given time $t$ can easily be found by integrating $0.5 \rho \mathrm{U}^{2}$ over $x$ from $-C_{s} t$ to infinity and is equal to $\rho_{0} C_{s}{ }^{3} t$. This calculates to $0.0024 \rho_{0}^{0.79} \mathrm{~T}^{2} \mathrm{t}\left(\mathrm{MJ} / \mathrm{cm}^{2}\right)$ and this matches the full physics simulation's opinion of the kinetic energy. Also that lower density profile within the rarefaction leads to a higher specific heat. This too can be easily found by doing a similar integral for $\rho$. We find that this lower density profile contributes an additional $\mu /(1-\mu)$ fraction of 
internal energy to that portion $\left(=\mathrm{C}_{\mathrm{S}} \mathrm{t} / \mathrm{x}_{\mathrm{F}}\right)$ of the heated matter overtaken by the rarefaction front. (The portion not overtaken is still at its original density.) For our value of $\mu$ this becomes $0.0011 \rho_{0}^{0.79} \mathrm{~T}^{2.4} \mathrm{t}\left(\mathrm{MJ} / \mathrm{cm}^{2}\right)$. These two effects together now lead to a corrected $\mathrm{E} / \mathrm{A}$ ( in $\mathrm{MJ} / \mathrm{cm}^{2}$ ) or the supersonic regime:

$$
\mathrm{E} / \mathrm{A}=0.0029 \mathrm{~T}^{3.55} \mathrm{t}^{.5} / \rho_{0}^{0.17}+0.0035 \rho_{0}^{0.79} \mathrm{~T}^{2.4} \mathrm{t} \quad \text { (full supersonic regime) }
$$

The solid curve in Fig. (7) is Eq. (2.3) calculated out to the high $\rho_{0}$ edge of the supersonic regime and largely reproduces the E/A full physics numerical simulation curve throughout the entire supersonic regime. While these additional energy sinks reduce the full "bonus" of being supersonic that Eq. (2.1) naively promises, we still note a nearly $20 \%$ reduction from the solid wall result.

Note too that in Fig. (7), Eq. (2.2) closely matches the full physics numerical simulation at the very high end of the initial-wall-density $x$-axis, deep in the subsonic regime. However, in the lower density part of the subsonic regime the simulations differ from the infinite density result. We believe that is due to the period of time early in the simulation when indeed the heat wave is supersonic and therefore less lossy. As the initial density, $\rho_{0}$, decreases, an increasingly longer early-time duration of supersonicity exists. We can correct for this by first finding $t_{\text {catch }}=0.17 \mathrm{~T}^{2.3} \rho_{0}{ }^{-1.9}$, the time when the rarefaction front, $C_{S} t$, catches up to the heat front (the $x_{F}(\mathrm{t})$ that precedes Eq. (3)). We then subtract the subsonic $\mathrm{E} / \mathrm{A}\left(\mathrm{t}=\mathrm{t}_{\text {catch }}\right)$ of Eq. (2.2) from $E / A(t)$ of Eq. (2.2) and add in its stead the supersonic $E / A\left(t=t_{\text {catch }}\right)$ of Eq. (2.3). For our gold parameters, the procedure outlined above leads to a simple expression for the correction:

$$
\mathrm{E} / \mathrm{A}\left(\mathrm{MJ} / \mathrm{cm}^{2}\right)=0.0058 \mathrm{~T}^{3.35} \mathrm{t}^{59}-0.002 \mathrm{~T}^{4.7} / \rho_{0}{ }^{1.1} \text { (full subsonic regime) }
$$

This result largely reproduces the E/A simulation curve throughout the entire subsonic regime, as seen in the dot-dashed curve of Fig. (7).

Since the minimum of E/A vs. $\rho_{0}$ occurs at densities low enough to be within the supersonic regime, we can easily take the $\mathrm{E} / \mathrm{A}$ derivative with respect to $\rho_{0}$ in Eq. (2.3) and find the optimal density, $\rho^{*}$ :

$$
\rho^{*}=0.17 \mathrm{~T}^{1.2} \mathrm{t}^{-0.5} \mathrm{~g}^{0.52} \mathrm{f}^{\prime-1.04} \text {. }
$$

In the above we explicitly included the scaling of the $\mathrm{Au}$ opacity and specific heat coefficients (the prime denotes them being scaled to their nominal values which were 
given above) so that this formula can be used more generally. Plugging this back into Eq. (2.3) gives us the minimum $\mathrm{E} / \mathrm{A}$ *

$\mathrm{E} / \mathrm{A}^{*}=0.0048 \mathrm{~T}^{3.35} \tau^{.59} \mathrm{f}^{0.68} \mathrm{~g}^{0.41}$.

Comparing this to the E/A of the very high density (solid and above) regime of Eq. (2.2) we see that they scale exactly the same way. Thus their ratio implies a universal (independent of $\mathrm{T}$ and $\mathrm{t}$ ) savings of $17 \%$ when the optimal $\rho^{*}$ is chosen as the initial wall density. (Also, self-consistently, $\rho^{*}$ "universally" falls within the supersonic regime).

Fig. (8) shows results from the $\mathrm{T}=1.25 \mathrm{heV}$ simulation set, wherein we plot the resultant E / A curves (normalized by their values at solid density) vs. initial density, for 3 pulse lengths varying from 1 to $64 \mathrm{~ns}$. We clearly see the "universal" nature the energy savings (vs. a solid wall) at the optimal density for each $t$ is the same value (of about $16 \%$ ), very close to our predictions.

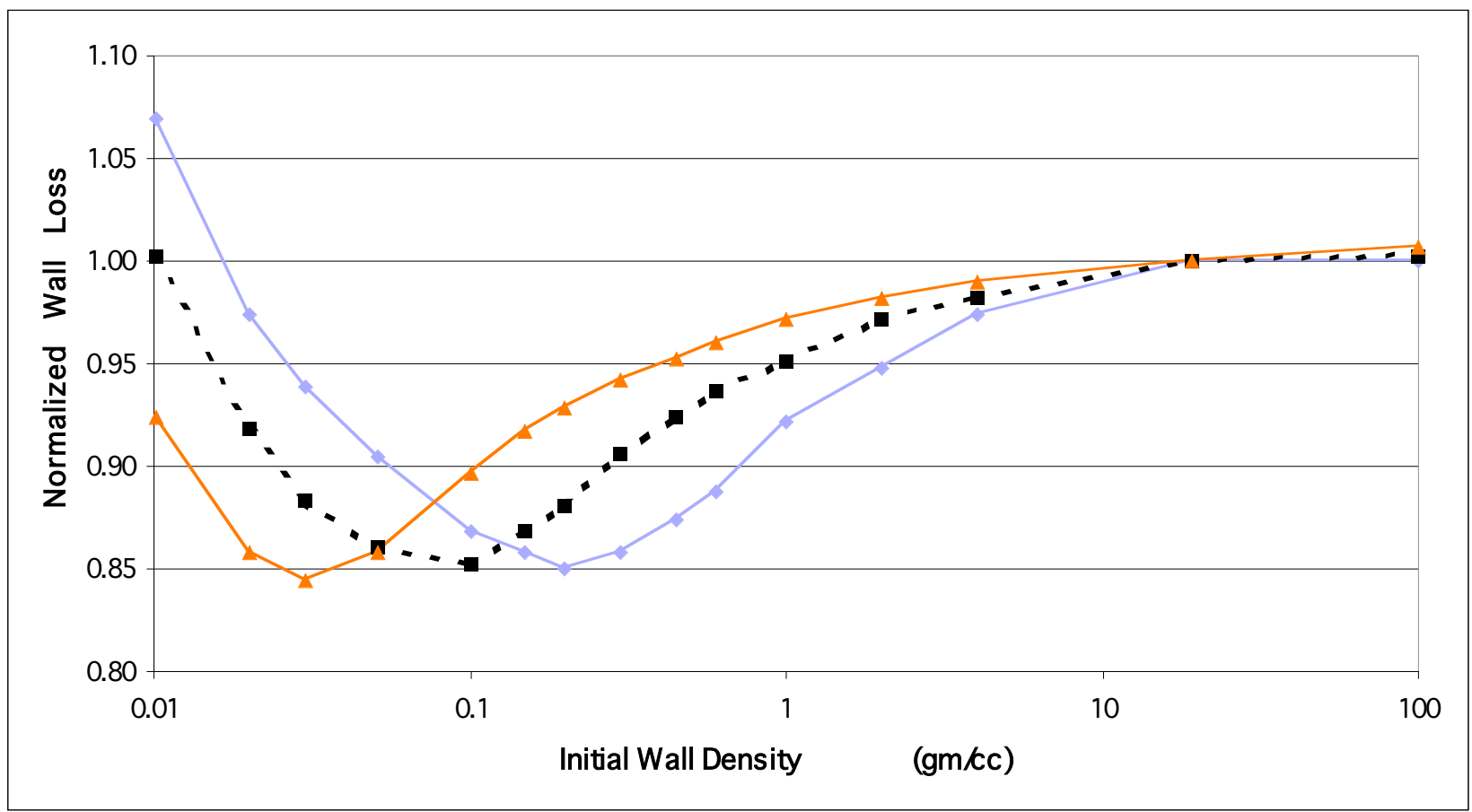

Figure 8 Simulated wall loss (normalized by wall loss at solid initial density) vs. initial wall density (gm/cc). Drive Conditions: $\mathrm{T}=125 \mathrm{eV}$; Duration t: triangles: $64 \mathrm{~ns}$. squares: 8 ns. diamonds: 1 ns 
Full 2-D Lasnex simulations also show that combining both schemes works best, namely foam cocktail hohlraums. This idea has been tried in detail for a heavy ion reactor scale hohlraum by Callahan (2000). It was optimized via tedious full 2-D simulations and a foam density of $0.1 \mathrm{gm} / \mathrm{cc}$ is arrived at. It is Au-Gd and as such $\mathrm{g}^{\prime}$ $=0.62, \mathrm{f}^{\prime}=1.04$, with $\mathrm{T}=2.5$ and $\mathrm{t}=8$, leading to our prediction of an optimal density of $0.13 \mathrm{gm} / \mathrm{cc}$ quite close to the optimized design value.

We have recently tested this foam wall as an energy saver concept using a cylinder of $\mathrm{Ta}_{2} \mathrm{O}_{5}$ made of either $4 \mathrm{gm} / \mathrm{cc}$ or $0.1 \mathrm{gm} / \mathrm{cc}$, each with a gold ring hit by the laser that served as the $\mathrm{x}$-ray source to drive the rest of the cylinder walls. They were performed by P. Young of LLNL at URLLE. A drooping pulse produced about a $100 \mathrm{eV}$ flat-topped drive. A Dante viewed the walls, and the $0.1 \mathrm{gm} / \mathrm{cc}$ foams were about $15 \%$ brighter in accord with 2-D simulations and in accord with the "source=sink" approach of this paper, when albedo effects are taken into account. More experiments are planned in a more fully enclosed hohlraum geometry. More work will need to be done to extend this idea to shaped pulses, for which perhaps graded density foams may have to be invoked.

\section{Hohlraums with Axial Shine Shields}

Another "trick" to save energy is to emplace axial shields (small Au disks) to block the capsule's view of the cold LEH, published by by Amendt et. al. (1996).. The laser beams enter the cylinder through entrance holes in the end cap as usual, but propagate through the outer "cold exterior"("ce", not to be confused here with "conversion efficiency") section, into the central "hot interior" ("hi") section of the hohlraum, and impinge on the walls there. The outer parts are cooler, and the aperture through which the radiation flows from the middle ("hi") to the ends ("ce") is the annulus between the on axis shine shield disk and the cylinder wall.

The LASNEX "observables" to be explained here are the $228 \mathrm{eV}$ for a hohlraum with no shine shields vs. a $241 \mathrm{eV}$ drive on capsule for one with shine shields. Why would a hohlraum that introduces about 500J of more wall loss via the shine shield disks, actually produce a hotter hohlraum rather than a cooler one? The answer (Rosen (1996)) to the paradox is essentially that we have created an inside out hohlraum, in which the central section is a "hot interior (hi) hohlraum", and 
drives the capsule. Indeed, the outer sections of these hohlraums are "cold exterior (ce) hohlraums", and are predicted by LASNEX to be only about $215 \mathrm{eV}$. We will derive all of these numbers presently, following the calculations of Rosen (1994b).

The cylinder is $0.8 \mathrm{~mm}$ in radius with a laser entrance hole (LEH) of $0.6 \mathrm{~mm}$ radius. The half length (from mid plane to end cap) of the can is $1.15 \mathrm{~mm}$. The shine shield is $0.3 \mathrm{~mm}$ in radius and placed on axis $0.55 \mathrm{~mm}$ from the midplane, (thus, 0.6 $\mathrm{mm}$ from the LEH). The capsule has a radius of $0.27 \mathrm{~mm}$. Thus the half-area of the hot interior hohlraum walls (from mid-plane to shine shield and including the shine shield disk area) Ahi is $3.04 \mathrm{~mm}^{2}$, of the cold exterior $\mathrm{A}_{\mathrm{ce}}, 4.2 \mathrm{~mm}^{2}$, and of the halfcapsule $A_{C}, 0.46 \mathrm{~mm}^{2}$. The LEH area $A_{L E H}$ is $1.13 \mathrm{~mm}^{2}$, and the annular area between shine shield and wall, Ahi-ce, through which the radiation flows from the hot interior to the cold exterior, is $1.73 \mathrm{~mm}^{2}$. A slight complication here is that some (about 33\%) of the laser energy is deposited along the cylinder wall between the axial position of the shine shield and the end of the can; namely there is some of the laser source in the cold exterior region. Nonetheless we can generalize our treatment of source=sink to account for this entire situation, and we will define EL to be made up of two parts ELhi and ELce for those amounts absorbed in the hi and ce regions respectively. In our case, for half of the incident $20 \mathrm{KJ}(=200 \mathrm{hJ})$ going into the half of the hohlraum being calculated, and at a conversion efficiency of $70 \%$, there is $1 / 2$ of $140 \mathrm{hJ}$ of $\mathrm{x}$-ray energy available. If $33 \%$ is created in the ce, then $47 \mathrm{hJ}$ is available in the hi and $23 \mathrm{hJ}$ in the ce. Defining $\mathrm{y}=\mathrm{T}_{\mathrm{ce}} / \mathrm{Th}_{\mathrm{hi}}$ and appealing as we have done throughout this paper to energy balance, and using our Au wall loss results for $\mathrm{k}=0.18$ as usual, we find the following:

The hot interior equation reads:

$$
\begin{aligned}
& \eta E_{\text {Lhi }}=\text { EWhi }+ \text { Ehi-ce }+E_{C} \quad \text { or } \\
& \eta E_{\text {Lhi }}=.4 \mathrm{Thi}^{3.3} \mathrm{Ahi}^{+} .6 \mathrm{Ahi} \text { ceThi }^{4}\left(1-\mathrm{y}^{4}\right)+.6 \mathrm{ACThi}^{4}
\end{aligned}
$$

while the cold exterior equation reads:

$$
\begin{aligned}
& \eta E_{\text {Lce }}+\text { Ehi-ce }=\text { EWce }+ \text { ELEH or } \\
& \eta E_{\text {Lce }}+.6 \text { Ahi-ceThi }{ }^{4}\left(1-\mathrm{y}^{4}\right)=.4 \mathrm{Thi}^{3.3} \mathrm{y}^{3.3} \mathrm{AS}+.6 \mathrm{~A}_{\mathrm{LEH}} \mathrm{Thi}^{4} \mathrm{y}^{4}
\end{aligned}
$$

Adding Eq. (2.81) to Eq. (2.7) we get:

$$
\eta \mathrm{EL}=\eta(\mathrm{ELhi}+\mathrm{ELce})
$$




$$
=.4 \mathrm{Thi}^{3.3} \mathrm{Ahi}\left(1+\left(\mathrm{A}_{\mathrm{ce}} / \mathrm{Ahi}\right) \mathrm{y}^{3.3}\right)+.6 \mathrm{ACT}_{\mathrm{Ti}}{ }^{4}\left(1+\left(\mathrm{A}_{\mathrm{LEH}} / \mathrm{AC}_{\mathrm{C}}\right) \mathrm{y}^{4}\right)
$$

Inserting the values for the areas (discussed above) Eq. (2.9) reduces to:

$$
70=1.27 \mathrm{Thi}^{3.3}\left(1+1.38 \mathrm{y}^{3.3}\right) \quad+.28 \mathrm{Thi}^{4}\left(1+2.5 \mathrm{y}^{4}\right)
$$

while Eq. (2.8) reduces to:

$$
23+1.04 \mathrm{Thi}^{4}\left(1-\mathrm{y}^{4}\right)=1.67 \mathrm{Thi}^{3.3} \mathrm{y}^{3.3}+.68 \mathrm{Thi}^{4} \mathrm{y}^{4}
$$

Equations (2.10) and (2.11) can be solved iteratively with a solution quickly converging to $\mathrm{Thi}=2.43$ and $\mathrm{y}=0.89$, namely $\mathrm{T}_{\mathrm{Ce}}=\mathrm{y} \mathrm{T}_{\mathrm{hi}}=2.16$. These values for the hot interior and cold exterior temperatures are in excellent agreement with LASNEX (2.41 \& 2.15 respectively). Moreover, had we considered a simple geometry (no shine shields) we would be solving:

$$
\begin{aligned}
& \eta E_{L}=\mathrm{EW}+\mathrm{ELEH}+\mathrm{EC} \quad \text { or } \\
& \eta \mathrm{EL}_{\mathrm{L}}=.4 \mathrm{~T}^{3.3} \mathrm{AW}+0.6 \mathrm{~T}^{4} \mathrm{AL}+.6 \mathrm{ACT}^{4}
\end{aligned}
$$

For the simple, no shine shield, geometry we find $A_{W}=6.7 \mathrm{~mm}^{2}$ and $A_{L}$ and $A_{C}$ are as above. Thus Eq. (2.12) reduces to:

$$
70=2.7 \mathrm{~T}^{3.3}+.95 \mathrm{~T}^{4}
$$

whose solution is $\mathrm{T}=2.31 \mathrm{heV}$, again in excellent agreement with the LASNEX result for no shine shield of $228 \mathrm{eV}$. The enhancement of temperature, taking the Lasnex numbers for example of 2.41 vs. 2.28, represent about a $20 \%$ energy savings. The reader can confirm this by putting those 2 values into Eq. (2.13) and getting 81 and $67 \mathrm{hJ}$ respectively.

This concept has been tested successfully and published. The drive increase in the hot interior hohlraum was measured via the decreased implosion time of a capsule therein. It was also noticed in those experiments that the axial shine shields provided yet another "knob" to control the symmetry of the illumination onto the capsule.

Thus we have presented in this lecture at least 3 ways to reduce wall losses, each by nearly $20 \%$, and each, in principle, can be used in conjunction with the other schemes, leading to an overall energy savings of $(0.8)^{3}$ or about 0.5 . This will allow NIF to operate quite far from its damage thresholds, and still provide the requisite drive to the capsule in the center of the hohlraum. We invite the reader to dream up any other energy savings schemes! 


\section{Lecture III: Applications of Hohlraums to ICF}

\section{Introduction:}

Space limitations here do not allow a complete description of the ICF problem. We refer the reader to Rosen (1999) for a rather complete tutorial on the subject. We in fact intend this Lecture III portion of this paper to be an auxiliary to that review, by completing / supplementing it with the next 2 sections. For completeness though, we'll just summarize the main points of that tutorial.

An ICF 1 GW reactor, with a 10\% efficient driver (supplying 6 MJ pulses @ 5 $\mathrm{Hz}$ to the target chamber) must have a target with gain $\mathrm{G}>100$. The inertial confinement time of an assembled fuel of radius $R$ is given by $R / 4 C_{S}$. The burn-up fraction $f_{B}$ of the fuel is given by $\rho R /(\rho R+70)$ in MKS units. We expect a target to operate optimally near $f_{B}=1 / 3$ or a $\rho R$ of 30 . With that fixed, and using "nature's bounty" the Q of DT being $3.410^{14} \mathrm{~J} / \mathrm{kg}$, we can see that to have the expected and containable output of $600 \mathrm{MJ}$ we must compress DT 1000 fold so that its mass will be about $510^{-6} \mathrm{~kg}$. This target has a momentum of $80 \mathrm{~kg} \mathrm{~m} / \mathrm{s}$ or the impact an average person would have walking into a wall at average walking speed, which is obviously quite containable.

A spherical implosion is the least stressing way to compress matter that much. A hohlraum allows for good symmetry for such an implosion as described at the very beginning of this article. There as well we described the method of implosion - basically a rocket. Thus there are 2 coupling efficiencies that get us from driver energy $E_{D}$ to the thermal energy of the assembled fuel: $\eta_{C}$ the coupling efficiency of the driver to thermal energy on the surface of the capsule. That hot gas is the exhaust of the rocket, which delivers a moving payload at efficiency $\eta_{\mathrm{H}}$. That kinetic energy is reconverted to the thermal energy of the assembled self-stagnating fuel.

If we had to heat that entire fuel assembly to $10 \mathrm{keV}$ to start the fusion process in earnest, it would require (1.5) $(10 \mathrm{keV})(4) / 5 \mathrm{AMU}=10^{12} \mathrm{~J} / \mathrm{kg}$, a factor $1 / 340$ less than the fusion pay-off $Q_{\text {DT }}$ quoted above. The ("4") of the above 
equation is due to the need to heat the deuteron, the triton, and the electron that comes with each. However, we only burn $1 / 3$ of that, and for a hohlraum typical coupling efficiencies are $\eta_{\mathrm{C}}=0.2$ and $\eta_{\mathrm{H}}=0.2$, thus the gain is only $G=(340)(0.2)$ $(0.2)(1 / 3)=5$, far too low for a reactor. The secret to high gain is to only heat a small central hot spot to $10 \mathrm{keV}$, and then let the alpha particle produced by the DT reaction stop within the surrounding fuel and do the heating. Typically that hot spot will have 0.1 of the surrounding dense fuel shell density, and its radius will be at about $\mathrm{R} / 2$. Thus it will have a negligible 0.01 of the mass of the fuel.

The requirements of a hot spot then, are to be $10 \mathrm{keV}$, and have a $\rho \mathrm{R}$ of 3 $\left(\mathrm{kg} / \mathrm{m}^{2}\right)$. That is the range of an alpha in a $10 \mathrm{keV}$ plasma. Moreover, by the $f_{B}$ formula, it implies the hot spot will burn 5\% of its fuel. That is precisely the amount needed to supply enough alphas to the first thin shell (a layer inside of the dense fuel which also has a $\rho \mathrm{R}$ of $3 \mathrm{~kg} / \mathrm{m}^{2}$ ) surrounding the hot spot to get it up to $10 \mathrm{keV}$, and thus launch the propagating thermonuclear burn-wave.

Energy is also required to compress the cold dense fuel to its high-density state in the shell that surrounds the hot spot. It is Fermi Degenerate matter, so its pressure will be $\mathrm{P}_{\mathrm{FD}}=2.210^{6} \alpha \rho^{5 / 3}(\mathrm{~Pa})$ and the energy required is $\mathrm{E}_{\mathrm{FD}}(\mathrm{J})=3.310^{6} \alpha$ $\rho^{2 / 3} M_{D T}(k g)$. Here $\alpha$ measures how far off the isentrope we are. We are now in a position to calculate the gain.

\section{Gain calculation: Conventional \& Fast Ignitor}

We begin with our incident pulse of $E_{D}=6 \mathrm{MJ}$. With the coupling and rocket efficiencies each of 0.2 means $E_{F}=0.24 \mathrm{MJ}$ is in the assembled fuel. Let us assume the hot spot radius is $50 \mu$, namely $\mathrm{R}_{\mathrm{HS}}=510^{-5} \mathrm{~m}$. This turns out to be near optimal. Since the hot spot requires $\rho \mathrm{R}$ of 3 , then $\rho_{\mathrm{HS}}$ must $=610^{4} \mathrm{~kg} / \mathrm{m}^{3}$. Then the mass of the hot spot is easily calculated to be $310^{-8} \mathrm{~kg}$, and its thermal energy (at the required $10 \mathrm{keV}$ ) is $\mathrm{E}_{\mathrm{HS}}=3.610^{4} \mathrm{~J}$. Its pressure $\mathrm{P}_{\mathrm{HS}}=2 \mathrm{n}_{\mathrm{i}} \mathrm{kT}$ (the 2 because of the electrons) is $4.610^{16} \mathrm{~Pa}$.

This self-stagnated assembly is isobaric, the hot spot pressure stops the cold fuel shell from imploding further, and pressures equilibrate $\mathrm{P}_{\mathrm{HS}}=\mathrm{P}_{\mathrm{C}}=\mathrm{P}_{\mathrm{FD}}$, which can then tells us what the cold density is: $\rho_{C}=1.610^{6} \mathrm{~kg} / \mathrm{m}^{3}$, indeed a 1000 fold denser than solid DT. We can plug that into the formula for the energy available for the 
cold dense shell: $\mathrm{E}_{\mathrm{FD}}=\mathrm{E}_{\mathrm{C}}=\mathrm{E}_{\mathrm{F}}-\mathrm{E}_{\mathrm{HS}}=2.410^{5}-3.610^{4}=2.0410^{5} \mathrm{~J}$, to obtain the mass of the shell, $\mathrm{M}_{\mathrm{C}}=4.810^{-6} \mathrm{~kg}$, in line with our assumption for the mass of the reactor target. We can then set $(4 / 3) \pi \rho_{C}\left(R^{3}-R_{H S}{ }^{3}\right)=M_{C}$ to find $R=9.510^{-5} \mathrm{~m}$, thus $\Delta R=R-$ $\mathrm{R}_{\mathrm{HS}}=4.510^{-5} \mathrm{~m}$ thus $\rho \Delta \mathrm{R}=70 \mathrm{~kg} / \mathrm{m}^{2}$ thus $\mathrm{f}_{\mathrm{B}}=70 /(70+70)=1 / 2$. Putting that altogether yields $G=\mathrm{f}_{B} \mathrm{M}_{C} \mathrm{Q}_{\mathrm{DT}} / \mathrm{E}_{\mathrm{D}}=(1 / 2)\left(4.810^{-6} \mathrm{~kg}\right)\left(3.410^{14} \mathrm{~J} / \mathrm{kg}\right) /\left(610^{6} \mathrm{~J}\right)=130$. Thus we have accomplished our "mission" of showing that ICF can in principle supply a gain 100 target to sustain a $1 \mathrm{GW}$ power reactor.

Before we leave this section let us compare this calculation with a fast ignitor scheme. In that approach we create a hot spot via an additional driver that creates the hot spot on the exterior of the assembled fuel. We do it quickly, so there is no pressure equilibrium between hot spot and cold dense fuel. Rather there is simply density equality (isochoric). As we shall see, this will allow the assembly to be larger but less dense, hence in general much less stressing to the implosion symmetry and stability requirements. So proceeding as before we note the requirements on the fast ignitor driver- it must supply $\mathrm{E}_{\mathrm{HS}}=3.610^{4} \mathrm{~J}$ in about a disassembly time of $10 \mathrm{ps}$, hence it must be of order 3 to $4 \mathrm{PW}$. Since now the densities are equal, $\rho_{\mathrm{C}}=610^{4} \mathrm{~kg} / \mathrm{m}^{3}$, so again solving for the mass of the cold assembly, $\mathrm{M}_{\mathrm{C}}=410^{-5}$, an order of magnitude larger than the conventional scheme!

With $M_{C}$ and $\rho_{C}$ known, we calculate $R=5.510^{-4}$, and $\Delta R=510^{-4}$ so $\rho \Delta R=30$ and thus $\mathrm{f}_{\mathrm{B}}=0.3$. The gain then is $\mathrm{G}=\mathrm{f}_{\mathrm{B}} \mathrm{M}_{\mathrm{C}} \mathrm{Q}_{\mathrm{DT}} / \mathrm{E}_{\mathrm{D}}=(0.3)\left(4.10^{-5} \mathrm{~kg}\right)\left(3.410^{14} \mathrm{~J} / \mathrm{kg}\right) /\left(610^{6} \mathrm{~J}\right)=$ 670, quite an improvement!

Another way to look at the advantage of a fast ignitor is to see that it gives large gain even for a smaller driver. Large drivers mean large initial capital investment expense- so the smaller the better. We invite the reader to re-do this calculation for $E_{\mathrm{D}}=1 \mathrm{MJ}$ not 6 . The optimal hot spot scales as $E_{\mathrm{D}}^{0.5}$, so take $\mathrm{R}_{\mathrm{HS}}=20 \mu$ this time. Find $\rho_{\mathrm{HS}}=1.510^{5} \mathrm{~kg} / \mathrm{m}^{3}=\rho_{\mathrm{C}}, \mathrm{M}_{\mathrm{HS}}=510^{-9} \mathrm{~kg}, \mathrm{E}_{\mathrm{HS}}=6 \mathrm{~kJ}, \mathrm{Ec}=.04 \mathrm{MJ}-6 \mathrm{~kJ}=$ $3.410^{4} \mathrm{j}$, thus $\mathrm{M}_{\mathrm{c}}=3.710^{-6} \mathrm{~kg}, \mathrm{R}=1.6110^{-4}, \rho \Delta \mathrm{R}=24, \mathrm{f}_{\mathrm{B}}=.256$, and $\mathrm{G}=312$. Obviously the great advantage of the fast ignitor is mitigated by the challenging physics of creating this externally driven hot spot.

Let us return then, to the conventional approach, and deal with one final topic: pulse shaping. 


\section{Pulse shaping}

If we analyze our conventional hot spot gain example from the previous section we can reach some important conclusions. We can ask: what was the required kinetic energy of the dense shell as it imploding in order to supply the thermal energy of the fuel when it stagnated and fully assembled? Normally we allow an "ignition margin" of a factor of 2 , so we set $(1 / 2) \mathrm{M}_{\mathrm{c}} \mathrm{V}_{\mathrm{imp}}{ }^{2}$ equal to $2 \mathrm{E}_{\mathrm{F}}$ which is almost $=2 \mathrm{E}_{\mathrm{C}}$. For a cold density of order $10^{6}$ as we calculated in our example, this then gives $V_{\text {imp }}=3.610^{5} \mathrm{~m} / \mathrm{s}$.

Other "external" requirements flow from this: Since the final fuel radius was about $100 \mu$, and a typical convergence ratio to get to those high densities is of order 30 , we get an initial radius of the capsule to be $3 \mathrm{~mm}$. Then an implosion time would be $\mathrm{R} / \mathrm{v}_{\mathrm{imp}}=10 \mathrm{~ns}$, and thus a power requirement of $600 \mathrm{TW}$, and an irradiance on target of order $600 \mathrm{TW} / 4 \pi(3 \mathrm{~mm})^{2}=510^{14} \mathrm{~W} / \mathrm{cm}^{2}$, or about $270 \mathrm{eV}$.

Another question to ask is what pressure is required to push the original shell to the required kinetic energy. We set $\mathrm{KE}=\mathrm{P} \Delta \mathrm{V}$, the "PdV" work done in implosion. Typically we start pushing at radius $R_{0}$, and stop when the shell is imploded to $r=R_{0} / 2$, since after that there is not much volume left to exploit for PdV work. The driver turns off and the shell coasts inward until stagnation. For a shell that starts at a thickness $\Delta R_{0}$, then our equation leads to $P=310^{13}\left(\Delta R_{0} / R_{0}\right)$. For stability reasons we avoid thin shells so we keep $\left(\Delta R_{0} / R_{0}\right)$ it to about $1 / 5$. This tells us that the pressure doing the pushing is $610^{12} \mathrm{~Pa}=60 \mathrm{Mbar}$.

The problem is we cannot apply that pressure to the original capsule! Consider the FD isentrope of solid DT. $P_{F D}=210^{6} \rho^{5 / 3}=1.410^{10} \mathrm{~Pa}$ for $\rho_{0}=200$ $\mathrm{kg} / \mathrm{m}^{3}$. Thus $60 \mathrm{MB}$ is way off the isentrope and would make it energetically very difficult to proceed with the implosion. Hence the need for pulse shaping.

If we compare the hugoniot relations for shocks vs. isentropic compression we learn a valuable lesson. For a jump of pressure $Y=P_{1} / P_{0}$ there will be a shocked density jump $X=\rho_{1} / \rho_{0}$. The Hugoniot relations tell us that $X=(4 Y+1) /(Y+4)$. Let us compare that to $X_{\text {isen }}=Y^{1 / \gamma}$ for $\gamma=5 / 3$. For $Y=1$ they are equal. For $Y=2, X=1.5$ and $X_{\text {isen }}=1.51$. For $Y=4, X=2.13$ and $X_{\text {isen }}=2.3$. For $Y=8, X=2.75$ and $X_{\text {isen }}=3.5$. For infinite $\mathrm{Y}, \mathrm{X}=4$ and $\mathrm{X}_{\mathrm{isen}}$ is infinite. So as long as the pressure jumps are less than 4 , the drift 
off of the isentrope via the sequential shock method will be less than $10 \%$. Thus our shape pulsing strategy should be clear. The first shock will necessarily be a strong one, hence $X=4$, hence the post-shock density will be $800 \mathrm{~kg} / \mathrm{m}^{3}$. Thus the $\mathrm{P}_{\mathrm{FD}}$ for that density will be $1.410^{11} \mathrm{~Pa}=1.4 \mathrm{Mb}$, and that should precisely be the magnitude of our first shock, to match that and stay on the FD isentrope. After that we launch 3 more shocks each 3.5 times bigger than the previous one. Our final pressure will be $(3.5)^{3}(1.4 \mathrm{Mb})=60 \mathrm{Mb}$ as required, and now the shell has compressed to the proper high density to remain on the FD isentrope as we push on it at $60 \mathrm{Mb}$ and accelerate it to the requisite implosion velocity of $3.610{ }^{5} \mathrm{~m} / \mathrm{s}$ on its way to a successful thermonuclear implosion. We must of course carefully time those shocks so that all the shocks coalesce at very near the inside of the frozen DT shell so that most of the fuel will remain cold Fermi Degenerate fuel.

With this explanation we also can understand the need for a frozen DT shell and not a gaseous target. If it were gas, its original density would be low, so even at $X=4$, the isentrope will have a very low $P$, and hence require much larger dynamic range on the low side for the driver to have. Moreover, as we must keep say $97 \%$ of the fuel on the cold FD isentrope, it means the shocks should coalesce at about 0.3 $R_{0}$. So now when we are ready for the final push to accelerate the cold (and now properly dense) fuel to the proper velocity we do not have very much volume left to do PdV work. Thus the final push must be much larger than $60 \mathrm{Mb}$, so we again stress the dynamic range of the driver this time on the high side! All told this is an untenable situation, and we must live with the complexities of frozen DT solid shelled targets.

This concludes our ICF overview. How coupling and gain scale with driver size is covered in Rosen (1999). Comparison with direct drive is also covered there. Direct drive has much better coupling efficiencies, but are more challenged on the hydro stability issue, which is beyond the scope of this review. It too is covered in Rosen (1999). Direct drive is making great strides in solving its stability issues, and in principle could have higher gains than indirect drive. HIF indirect drive targets have no LEH, so their coupling efficiency is better than laser driven hohlraums so they can reach a gain 100 at about 3 MJ driver. They show great promise as well since the drivers are high efficiency and in principle high reliability. 
In summary we have shown the principles of ICF hohlraums. We showed how they naturally have an increase of efficiency of coupling initial laser energy to absorption by the fuel capsule as we increase in scale from Nova to NIF to reactors. This helps motivate the predictions of the complex LASNEX simulations that NIF will achieve moderate gains of 10-20, and reactors will achieve higher gains allowing them to be competitive energy sources for the next century.

We gratefully acknowledge the warm hospitality of St. Andrews University and the NATO sponsored Scottish University Summer School 60 under the directorship of Dr. Dino Jarosyznski and ably assisted by Dr. Alan Cairns and Ms. Giselle Smith. Useful conversations with our LLNL theory and design colleagues J. Hammer, O. Jones, L. Suter, J. Edwards, A. Szoke, J. Castor, S. MacLaren, R. Thiessen, and P. Amendt, as well as with our AWE colleagues B. Thomas, P. Thompson, M. Stevenson and S. McAlpin. Our experimental collaborators are also gratefully acknowledged: J. Schein, P. Young, E. Dewald, N. Landen, S. Glenzer, K. Campbell, R. Turner, R. Kauffman, C. Darrow, H. Kornblum, J. Porter, T. Orszechowski, and W. Hsing. The computational and data base assistance was ably provided by G. Zimmerman, J. Harte, D. Bailey, M. Marinak, J. Albritton, B. Goldstein, and B. Wilson, for which we are grateful. Target fabrication work by R. Wallace, J. Gunther et al of LLNL's ICF program and J. Kass, A. Hamza et al of LLNL's C\&MS department were invaluable, as was the target fabrication efforts of colleagues at GA: H. Wilkens, A. Nikroo, J. Kaae, T. Back, J. Kilkenny, and M. Campbell. The collaborations with our Sandia colleagues R. Olson, G. Rochau, and R. Leeper are also appreciated. The encouragement and support of B. Hammel, J. Lindl, E. Moses, G. Miller, C. Verdon, B. Goodwin, and M. Anastasio are also gratefully acknowledged. The privilege of having J. Nuckolls as a mentor is hereby acknowledged as being valuable beyond measure. This work as performed under the auspices of the US. Department of Energy, by the Lawrence Livermore National Laboratory under contract W-7405-ENG-48.

This work was performed under the auspices of the U.S. Department of Energy by University of California, Lawrence Livermore National Laboratory under contract W-7405-Eng-48. 


\section{References:}

Amendt $\mathrm{P}$ et al, 1996, Novel Symmetry Tuning in Nova Hohlraums Using Axial Gold Disks, Physics of Plasmas 34166.

Callahan D and Tabak M, 2000, Progress in target physics and design for heavy ion fusion, Phys. Plasmas 72083.

Hammer J H and Rosen M D, 2003, Exact solutions to the diffusive radiation transport equations, Physics of Plasmas 101829.

Jones O S et al 2004 Measurement of the Absolute Hohlraum Wall Albedo under Ignition Foot Drive Conditions, Physical Review Letters 93065002.

Kauffman R L et al, 1994, High Temperatures in Inertial Confinement Fusion Radiation Cavities Heated with $0.35 \mu$ Light, Physical Review Letters 732320.

Marshak R E, 1958 , Effects of Radiation on Shock Wave Behavior, Phys. Fluids 124.

Olsen $\mathrm{R}$ et al, 2003, Time and spatially resolved measurements of x-ray burnthrough and re-emission in Au and Au:Dy:Nd foils, Rev. Sci. Instr. 742186.

Orzechowski T J, et. al, 1996, The Rosseland Mean Opacity of a Mixture of Gold and Gadolinium at High temperatures, Phys. Rev. Lett. 773545.

Rosen M D, 1979, Scaling law for radiation temperature, Laser Program Annual Report (1979), Lawrence Livermore National Laboratory, Livermore, CA, UCRL-50055-79, pp. 2-37 to 2-46 (unpublished). This work was extensively quoted and presented in J. Lindl, Phys. Plasmas, 2, 3933, (1995).

Rosen M D, Lindl J D, and Kilkenny J D, 1994a, Recent results on Nova, Journal of Fusion Energy 13155.

Rosen M D, 1994b, Scaling laws for non-conventional hohlraums, Bulletin of the American Physical Society 391684.

Rosen M D, 1996, The science applications of the high-energy density plasmas created on the Nova laser, Phys. Plasmas 31803.

Rosen M D, 1999, The physics issues that determine ICF target gain and driver requirements: A tutorial, Phys. Plasmas 61690.

Rosen M D and Hammer J H, 2005, Foam walled Hohlraums, submitted to Phys. Rev E.

Zimmerman G B and Kruer W L, 1975, The LASNEX simulation code, Comments in Plasma Physics and Controlled Fusion 285. 